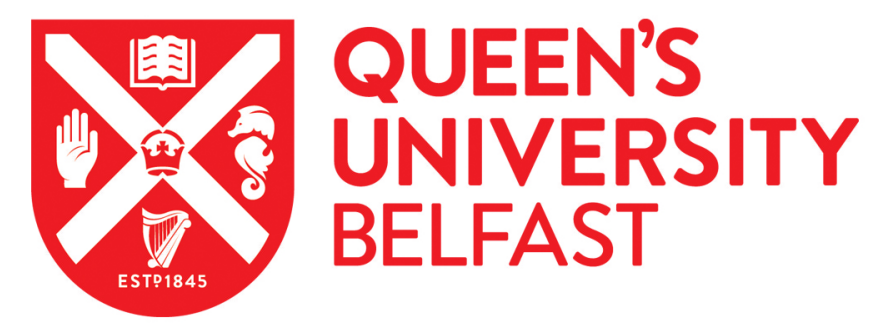

\title{
Establishing Earth's Minimoon Population through Characterization of Asteroid 2020 CD3
}

Fedorets, G., Micheli, M., Jedicke, R., Naidu, S. P., Farnocchia, D., Granvik, M., Moskovitz, N., Schwamb, M. E., Weryk, R., Wierzchoś, K., Christensen, E., Pruyne, T., Bottke, W. F., Ye, Q., Wainscoat, R., Devogèle, M., Buchanan, L. E., Djupvik, A. A., Faes, D. M., ... Smith, A. B. (2020). Establishing Earth's Minimoon Population through Characterization of Asteroid 2020 CD3. Astronomical Journal, 160(6). https://doi.org/10.3847/1538$3881 / a b c 3 b c$

Published in:

Astronomical Journal

Document Version:

Peer reviewed version

Queen's University Belfast - Research Portal:

Link to publication record in Queen's University Belfast Research Portal

Publisher rights

(c) 2020. The American Astronomical Society

This work is made available online in accordance with the publisher's policies. Please refer to any applicable terms of use of the publisher.

General rights

Copyright for the publications made accessible via the Queen's University Belfast Research Portal is retained by the author(s) and / or other copyright owners and it is a condition of accessing these publications that users recognise and abide by the legal requirements associated with these rights.

Take down policy

The Research Portal is Queen's institutional repository that provides access to Queen's research output. Every effort has been made to ensure that content in the Research Portal does not infringe any person's rights, or applicable UK laws. If you discover content in the Research Portal that you believe breaches copyright or violates any law, please contact openaccess@qub.ac.uk. 
Draft version November 23, 2020

Typeset using LATEX default style in AASTeX63

\title{
Establishing Earth's minimoon population through characterization of asteroid $2020 \mathrm{CD}_{3}$
}

\author{
Grigori Fedorets, ${ }^{1}$ Marco Micheli, ${ }^{2,3}$ Robert Jedicke, ${ }^{4}$ Shantanu P. Naidu, ${ }^{5}$ Davide Farnocchia, ${ }^{5}$ \\ Mikael Granvik, ${ }^{6,7}$ Nicholas Moskovitz, ${ }^{8}$ Megan E. Schwamb, ${ }^{1}$ Robert Weryk, ${ }^{4}$ Kacper Wierzchoś,${ }^{9}$ \\ Eric Christensen, ${ }^{9}$ Theodore Pruyne, ${ }^{9}$ William F. Bottke, ${ }^{10}$ Quanzhi Ye, ${ }^{11}$ Richard Wainscoat, ${ }^{4}$ \\ Maxime Devogèle, ${ }^{8}$ Laura E. Buchanan, ${ }^{1}$ Anlaug Amanda Duupvik, ${ }^{12}$ Daniel M. Faes, ${ }^{13}$ Dora Föhring, ${ }^{4}$ \\ Joel Roediger, ${ }^{14}$ Tom Seccull, ${ }^{13}$ And Adam B. Smith ${ }^{13}$ \\ ${ }^{1}$ Astrophysics Research Centre, School of Mathematics and Physics, Queen's University Belfast, Belfast BT7 1NN, UK \\ ${ }^{2}$ ESA NEO Coordination Centre, Largo Galileo Galilei, 1, 00044 Frascati (RM), Italy \\ ${ }^{3}$ INAF - Osservatorio Astronomico di Roma, Via Frascati, 33, 00040 Monte Porzio Catone (RM), Italy \\ ${ }^{4}$ University of Hawai' $i$, Institute for Astronomy, 2680 Woodlawn Drive, Honolulu, Hawai'i, 96822, USA \\ ${ }^{5}$ Jet Propulsion Laboratory, California Institute of Technology, Pasadena, CA, 91109, USA \\ ${ }^{6}$ Department of Physics, P.O. Box 64, 00014 University of Helsinki, Finland \\ ${ }^{7}$ Asteroid Engineering Laboratory, Onboard Space Systems, Luleå University of Technology, Box 848, 98128 Kiruna, Sweden \\ ${ }^{8}$ Lowell Observatory, 1400 W Mars Hill Road, Flagstaff, AZ 86001, USA \\ ${ }^{9}$ The University of Arizona, Lunar and Planetary Laboratory, 1629 E. University Blvd., Tucson, AZ 85721, USA \\ ${ }^{10}$ Department of Space Studies, Southwest Research Institute, 1050 Walnut Street, Suite 300, Boulder, CO 80302, USA \\ ${ }^{11}$ Department of Astronomy, University of Maryland, College Park, MD 20742, USA \\ ${ }^{12}$ Nordic Optical Telescope, Apartado 474, E-38700 Santa Cruz de La Palma, Santa Cruz de Tenerife, Spain \\ ${ }^{13}$ Gemini Observatory/NSF's NOIRLab, 670 N. A'ohoku Place, Hilo, Hawai'i, 96720, USA \\ ${ }^{14}$ National Research Council of Canada, Herzberg Astronomy and Astrophysics Research Centre, 5071 West Saanich Road, Victoria, BC \\ V9E 2E7, Canada
}

\begin{abstract}
We report on our detailed characterisation of Earth's second known temporary natural satellite, or minimoon, asteroid $2020 \mathrm{CD}_{3}$. An artificial origin can be ruled out based on its area-to-mass ratio and broad-band photometry, which suggest that it is a silicate asteroid belonging to the $\mathrm{S}$ or $\mathrm{V}$ complex in asteroid taxonomy. The discovery of $2020 \mathrm{CD}_{3}$ allows for the first time a comparison between known minimoons and theoretical models of their expected physical and dynamical properties. The estimated diameter of $1.2_{-0.2}^{+0.4}$ meters and geocentric capture approximately a decade after the first known minimoon, $2006 \mathrm{RH}_{120}$, are in agreement with theoretical predictions. The capture duration of $2020 \mathrm{CD}_{3}$ of at least 2.7 years is unexpectedly long compared to the simulation average, but it is in agreement with simulated minimoons that have close lunar encounters, providing additional support for the orbital models. $2020 \mathrm{CD}_{3}$ 's atypical rotation period, significantly longer than theoretical predictions, suggests that our understanding of meter-scale asteroids needs revision. More discoveries and a detailed characterisation of the population can be expected with the forthcoming Vera C. Rubin Observatory Legacy Survey of Space and Time (LSST).
\end{abstract}

\section{INTRODUCTION}

Asteroids and comets can be temporarily captured by planets as natural satellites. Theoretical models (Granvik et al. 2012; Fedorets et al. 2017) predict that the Earth is also surrounded by a cloud of such temporarily captured asteroids, colloquially called minimoons. The largest minimoon captured at any given time is one meter in diameter, while larger bodies are captured less frequently.

Minimoons possess a number of attributes which make them objects of particular interest. As they spend an extended amount of time in the vicinity of the Earth, they can provide several windows of opportunity to obtain observations of the little studied population of meter-class asteroids. The systematic discovery and population statistics of meter to decameter-class minimoons, a sub-population of the near-Earth objects (NEO), could resolve existing disagreements 
between extrapolations of different NEO size-frequency distribution models to this size range - i.e. those based, on telescopic observations (e.g., Rabinowitz et al. 2000; Harris \& D'Abramo 2015; Granvik et al. 2016; Tricarico 2017) and those based on on bolide data (Brown et al. 2002; Brown et al. 2013). Also, due to their relatively long capture duration, accessibility, and small size, minimoons are viable targets for taking the first practical steps in the emerging field of asteroid in situ resource utilisation (Granvik et al. 2013; Jedicke et al. 2018a). So far, the primary obstacle for organizing their study has been a lack of observational evidence supporting the existence of a minimoon population to the extent predicted by the models.

Until 2020, only one minimoon (2006 $\mathrm{RH}_{120}$; Bressi et al. 2008; Kwiatkowski et al. 2009) had been discovered. The second known minimoon, $2020 \mathrm{CD}_{3}$, was discovered on 2020 February 15.51 UT by the Catalina Sky Survey (CSS; Christensen et al. 2018) $1.5 \mathrm{~m}$ telescope on Mt. Lemmon (Minor Planet Center 2020). One day later, an alert automatically sent out by the NASA Jet Propulsion Laboratory's Scout system (Farnocchia et al. 2015a; Farnocchia et al. 2016) announced that it was likely temporarily captured in the Earth-Moon system. Discovering an object on a geocentric orbit always raises suspicion of an artificial origin, but during the 2.5 weeks following its discovery $2020 \mathrm{CD}_{3}$ could not be linked to any known artificial object nor could a natural origin be ruled out. On 2020 February 26 the Minor Planet Center (MPC) therefore added $2020 \mathrm{CD}_{3}$ to the catalogue of asteroids as a temporarily-captured object with a request for further follow-up observations to establish its nature (Minor Planet Center 2020).

The nominal solution for the area-to-mass ratio of $2020 \mathrm{CD}_{3}$ - calculated from the solar radiation pressure signature on the orbital solution, and a diagnostic quantity for distinguishing between natural and artificial objects (Jedicke et al. 2018a) - decreased during the two weeks after discovery (Fig. 1), indicating that it might be a natural object. To characterize the potential minimoon, we obtained high-precision astrometric follow-up observations in FebruaryMay 2020 with the Nordic Optical Telescope (NOT), Canada-France-Hawai'i Telescope (CFHT), Lowell Discovery Telescope (LDT), University of Hawai'i $2.2 \mathrm{~m}$ telescope (UH2.2) and the Calar Alto Schmidt telescope; broad-band photometric observations from Gemini North; and rotational lightcurve observations with LDT. In addition, a search for pre-discovery detections with the Pan-STARRS surveys (Chambers et al. 2016), Zwicky Transient Facility (Bellm et al. 2019; Masci et al. 2019), Catalina Sky Survey (Christensen et al. 2018), and Chinese Near-Earth Object Survey Telescope (Zhao et al. 2007) was performed.

In this work, we provide a detailed characterization of the physical properties and orbital evolution of $2020 \mathrm{CD}_{3}$. We also discuss its detectability and assess the possibility of its lunar origin. We describe the observations and precovery attempts in detail in Sect. 2; outline the data reduction and methods for physical characterization and orbit computation in Sect. 3; present the results and discuss the implications in Sect. 4; and offer our conclusions in Sect. 5.

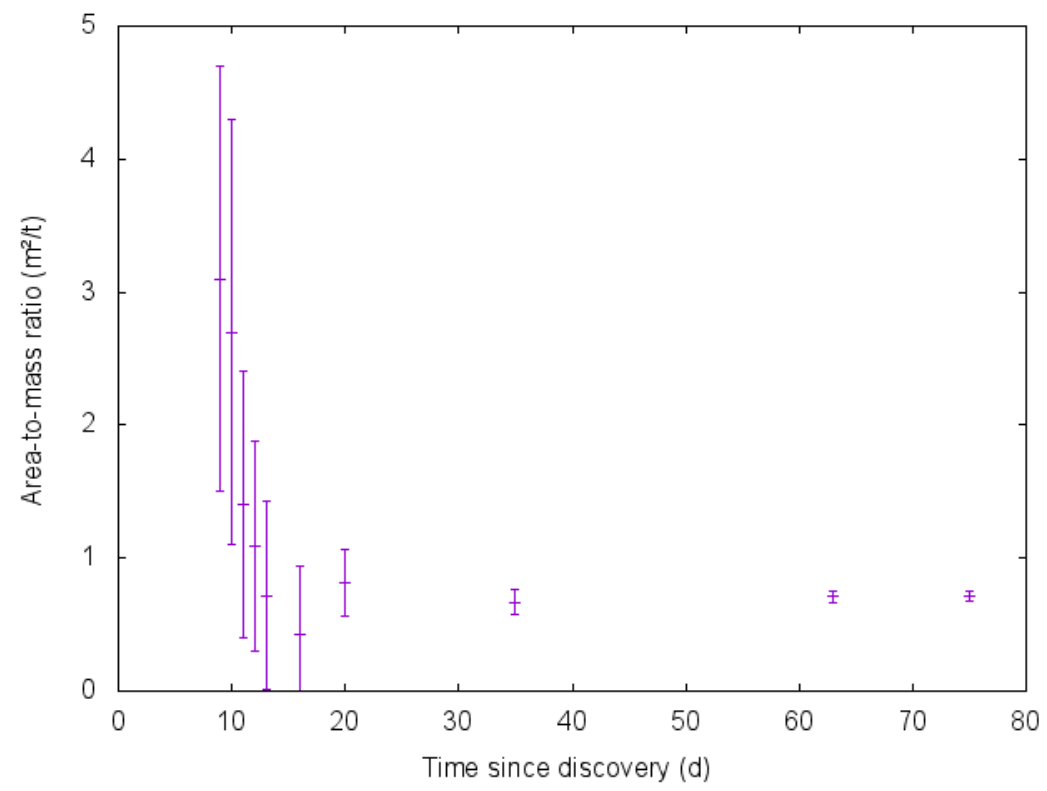

Figure 1. The evolution of the detection of the zero-albedo area-to-mass ratio with $1 \sigma$ errorbars as the function of the length of the observational arc. 


\section{DATA ACQUISITION}

\subsection{Instruments and observations}

An overview of all instruments used in this analysis is provided in Appendix Table 1. $2020 \mathrm{CD}_{3}$ was discovered on 2020 February 15.51 UT by the Catalina Sky Survey (CSS; Christensen et al. 2018) $1.5 \mathrm{~m}$ telescope on Mt. Lemmon (MPC observatory code G96). Upon discovery, the object was favorably placed near the ecliptic plane $\sim 45^{\circ}$ east of opposition. The discovery image sequence consisted of four $30 \mathrm{~s}$ exposures, with $\sim 7$ minutes separation between each successive image, that were inspected soon after the final image by two observers and submitted to the MPC as a new NEO candidate. After the object was placed on the MPC's NEO Confirmation Page, additional same-night follow-up observations were performed with the same telescope that was used to discover $2020 \mathrm{CD}_{3}$.

Multiple broadband photometric imaging was performed on 2020 February 24 with the $8.1 \mathrm{~m}$ Frederick C. Gillett Gemini North Telescope located on Maunakea, Hawai'i, USA. The Gemini Multi-Object Spectrograph (GMOS; Hook et al. 2004) observations consisted of three $r^{\prime} g^{\prime} i^{\prime}$ sequences with the G0301, G0303, and G0302 filters, taken in photometric conditions with Image Quality 85 (1.05" full zenith corrected seeing) or better seeing. The telescope was tracked non-sidereally at $2020 \mathrm{CD}_{3}$ 's rate of motion, thereby maintaining its stellar point spread function (PSF) for photometry but elongating the reference field stars. We also obtained sidereally tracked images in the three filters immediately before and after the non-sidereal tracking of $2020 \mathrm{CD}_{3}$ in order to perform absolute photometry.

To obtain $2020 \mathrm{CD}_{3}$ 's photometric lightcurve we employed the Large Monolithic Imager (LMI) on the $4.3 \mathrm{~m}$ Lowell Discovery Telescope (LDT, G37) for approximately 1 hour on 2020 February 27 UTC. Exposures were taken with $30 \mathrm{~s}$ integrations using a broad band $V R$ filter that provides high throughput between approximately 500 and $700 \mathrm{~nm}$. LMI was binned $3 \times 3$ for an effective plate scale of $0.36 " /$ pixel and the telescope was tracked at the non-sidereal rates of the target. On several later occasions we used LDT/LMI to obtain astrometry with a similar technique but without any filters.

The 3.6 m Canada France Hawaii Telescope (CFHT, 568) on Maunakea, Hawai'i, USA, was used to obtain astrometry using non-sidereal tracking with exposures of up to $120 \mathrm{~s}$ in gri-band MegaCam images with no pixel binning. MegaCam has $0.187^{\prime \prime}$ pixels allowing for precise astrometric measurements under good seeing conditions.

Astrometric observations were also made with the Alhambra Faint Object Spectrograph and Camera (ALFOSC) at the $2.5 \mathrm{~m}$ Nordic Optical Telescope (NOT, Z23) at the Roque de los Muchachos Observatory, La Palma, Canary islands, Spain. The exposures were tracked non-sidereally on $2020 \mathrm{CD}_{3}$. Each image's exposure time was set equal to the time it would take for $2020 \mathrm{CD}_{3}$ to move at most one stellar FWHM on the sky. Most of the $2020 \mathrm{CD}_{3}$ detections had $S / N \geq 15$ but the last observations reached only $S / N \sim 5$ as the target reached the detection threshold. The observations were performed without any filters with $4 \times 4$ pixel binning.

The University of Hawaii $2.2 \mathrm{~m}$ (UH2.2, 568) telescope was used for astrometric observations with non-sidereal tracking at $2020 \mathrm{CD}_{3}$ 's apparent rates of motion in unfiltered $300 \mathrm{~s}$ exposures. Additional astrometric observations were extracted from dedicated early observations obtained with the Calar Alto Schmidt telescope (Z84) in Spain. The detections were obtained from a set of short sidereally tracked frames, stacked with respect to the known motion of the object.

\subsection{Search for pre-discovery detections}

The image archives for several survey telescopes were searched for pre-discovery observations of $2020 \mathrm{CD}_{3}$ by generating an ephemeris for each exposure and visually examining any potential matches. The $1.8 \mathrm{~m}$ Pan-STARRS1 telescope (F51) has an extensive archive dating back to 2010 (Chambers et al. 2016) and is sensitive to $V \sim 23$ but no detections were found. The Zwicky Transient Facility (ZTF, I41) is an ongoing wide-field optical survey using the $1.2 \mathrm{~m}$ Palomar Oschin Schmidt telescope and has been in operation since 2018 (Bellm et al. 2019; Graham et al. 2019; Masci et al. 2019). No detections were found in its Data Release 3 (DR3) archive that extends from March 2018 to the end of December 2019.

The Chinese NEO Survey Telescope (CNEOST, D29; Zhao et al. 2007) is a $1.0 \mathrm{~m}$ Schmidt telescope at Xuyi, Jiangsu, China, equipped with a $3^{\circ} \times 3^{\circ}$ camera. We searched images taken between January 2018 and May 2019 (when the telescope went offline for hardware upgrades) but did not find any matching fields. Lastly, we checked all the telescopes used by the Catalina Sky Survey for pre-discovery opportunities and found only two suitable fields imaged by the Mt. Lemmon telescope (G96) on 2019 November 9 and 2019 January 24, close to times when the object was expected to be at perigee and therefore relatively bright. Significant trailing losses, the spreading of the light from the target over many pixels due to its motion during an exposure, combined with non-optimal sky conditions, prevented a detection in 
both images. In summary, the signals in the possible images were mostly smeared by trailing losses, and no detections were found from any of the mentioned surveys.

\section{DATA REDUCTION AND CALCULATIONS}

\subsection{Astrometric data reduction}

Due to the different observing strategies and capabilities of each instrument/telescope combination, each image set was astrometrically analyzed with different techniques. In some cases, a direct measurement on individual frames was possible by fitting $2020 \mathrm{CD}_{3}$ 's detection to a stellar PSF or trail. In other cases, especially later in the apparition, we stacked multiple frames at $2020 \mathrm{CD}_{3}$ 's (often rapidly changing) rates of motion to achieve sufficient SNR for a measurable detection. We carefully estimated our formal astrometric uncertainty taking into account contributions from the object's SNR (often dominant), but also from the astrometric solution, now typically negligible thanks to the Gaia DR2 catalogue (Gaia Collaboration et al. 2016, 2018; Lindegren et al. 2018), to which all the astrometry was calibrated. For all instruments used in the analysis, an assessment of the timing accuracy was also included. In most cases, a conservative timing uncertainty of one second was assumed. When timing biases were suspected, we only included the cross-track component of the astrometric position in the astrometric fit, and deweighted the along-track direction. All acquired and remeasured astrometry is provided in Appendix Table 2.

The peculiarities of $2020 \mathrm{CD}_{3}$ 's outgoing trajectory and, in particular, its low relative velocity with respect to Earth, kept the object at small geocentric distances for many weeks after discovery. As a result, most of the astrometric coverage was obtained when topocentric parallax was significant, and it is essential to know the precise and accurate location of the observing telescope, ideally to within a few meters in the Gaia catalog era. We therefore dedicated significant effort to obtain accurate coordinates and/or codes for all the telescopes we used to extract observations of $2020 \mathrm{CD}_{3}$.

\subsection{Photometric data reduction}

The raw GMOS-N data frames were reduced using standard techniques with the Gemini DRAGONS Python package (Data Reduction for Astronomy from Gemini Observatory North and South, AURA Gemini Observatory-Science User Support Department 2018). Nightly bias frames and twilight flats from the several nights surrounding the observations were used to create the master bias and flat-fields. The DAOPHOT software package (Stetson 1987), embedded in the Image Reduction and Analysis Facility (IRAF, Tody 1986, 1993), was used to perform aperture photometry for all the GMOS images. The photometry was calibrated to the Sloan Digital Sky Survey (SDSS) photometric system $\left(g^{\prime}, r^{\prime}\right.$ and $i^{\prime}$, Fukugita et al. 1996) with the SDSS Data Release catalog 12 (Alam et al. 2015), accessed through the SkyServer platform. The resulting individual measurements and errors of GMOS photometry is provided in Appendix Table 3. The resulting magnitudes in each filter are mean values of individual measurements with respective filters. That way we diminish the effect of the brightness variations induced by the rotation of the asteroid.

The set of images obtained with LDT/LMI for the lightcurve were reduced using standard bias subtraction and flat field correction from facility dome flats. $2020 \mathrm{CD}_{3}$ 's photometry was measured using the Photometry Pipeline (Mommert 2017). This pipeline extracted sources with SourceExtractor using a 3 pixel (1.08") aperture (Bertin \& Arnouts 1996), astrometrically registered the images based on the Gaia DR2 catalog (Gaia Collaboration et al. 2018), and then determined the zero point calibration for each image by referencing to approximately 50 field stars from the Pan-STARRS DR1 catalog (Flewelling et al. 2016). The photometric calibration was performed by tying the $V R$-images to the Pan-STARRS1 $r_{\mathrm{P} 1}$ band. This technique introduces errors in the absolute photometric calibration as the bands are not identical but they are significantly smaller than the typical uncertainty $(\sim 0.1-0.2$ magnitude $)$ on the individual measurements. All data points for the lightcurve are provided in Appendix Table 4.

\subsection{Calculations of area-to-mass ratio, albedo, density and phasecurve}

The astrometric data shows that the motion of $2020 \mathrm{CD}_{3}$ is significantly affected by solar radiation pressure. Establishing its signature with a $3 \sigma$ detection in about three weeks is $2-3$ times faster than similar analyses in the past. The evolution of the development of the radiation pressure as a function of time is presented in Fig. 1. This improvement is due to the enhanced precision and accuracy of the astrometry enabled by the Gaia DR2 catalogue (Gaia Collaboration et al. 2018; Lindegren et al. 2018) which permits measuring individual ground-based positions with $0.05^{\prime \prime}$ accuracy.

In what follows, we interpret the non-gravitational acceleration as a result of Solar radiation pressure. Following Farnocchia et al. (2015b), we modelled solar radiation pressure perturbation as a purely radial acceleration $A_{1} / r^{2}$, 
where $r$ is the heliocentric distance. The off-radial components, $A_{2}$ and $A_{3}$, of the Marsden non-gravitational force model (Marsden 1969; Marsden et al. 1973) do not play a significant role in the albedo-density modelling, unlike for the orbital evolution. The $A_{1}$ parameter is proportional to the area-to-mass ratio $A / m$ and therefore can provide useful constraints on the physical properties of the object and discern between a natural and artificial origin. For a spherical object,

$$
A_{1}=A / m\left(1+\frac{4}{9} A\right) \frac{G_{S}}{c}, A / m=\frac{3}{2 D \rho},
$$

where $A$ is the Bond albedo, $G_{S}$ is the solar constant, $c$ is the speed of light, $D$ the effective diameter, and $\rho$ the density (Vokrouhlický \& Milani 2000; Mommert et al. 2014a). We note that this formulation does not take into account the Yarkovsky effect (cf. Vokrouhlický 1998), which could contribute to 10-20\% of the total radial non-gravitational acceleration (e.g., Chesley et al. 2014). Therefore, our calculation is an upper bound estimate of $A / m$.

The effective diameter $D$, absolute magnitude $H$, and geometric albedo $p$ are related by (Pravec \& Harris 2007):

$$
D=1329 \mathrm{~km} \frac{10^{-0.2 H}}{\sqrt{p}},
$$

while the Bond albedo $A$ is the product of the geometric albedo $p$ and the phase integral $q$,

$$
A=p q=p\left(0.009082+0.4061 G_{1}+0.8092 G_{2}\right),
$$

where we have expressed the phase integral $q$ in terms of the $G_{1}$, and $G_{2}$ photometric parameters (Muinonen et al. 2010).

\subsection{Orbit computation}

We used a Monte Carlo approach to analyze $2020 \mathrm{CD}_{3}$ 's past trajectory. We generated 1000 synthetic sets of orbital elements and area-to-mass ratios by sampling the uncertainty region as calculated from the fit to the astrometry. We modelled the solar radiation perturbation using all three coefficients $\left(A_{1}, A_{2}, A_{3}\right)$ of the Marsden non-gravitational model (Marsden 1969; Marsden et al. 1973). Given the size of $2020 \mathrm{CD}_{3}$ and its unknown shape, unlike for the calculation of the area-to-mass ratio, for orbit computation the off-radial components of the solar radiation pressure signature are significant on the timescale of the capture duration. We integrated each synthetic object backwards from 2020 until the object had been captured into the Earth-Moon system. The date of the first perigee within 1 lunar distance (LD) after the insertion into the Earth-Moon system is used as a proxy for the capture date.

Several synthetic objects's orbits were consistent with a lunar origin and their distribution at launch from the Moon's surface is provided in Appendix Fig. 6 assuming that the Moon is a sphere of radius $1737 \mathrm{~km}$. In order to trace the possible origin of $2020 \mathrm{CD}_{3}$ from the Moon, we mapped the outbound trajectories of the samples originating from the Moon on the Lunar surface. We computed the state vectors of the samples when leaving the Moon's surface and transformed them into the Lunar mean Earth/polar axis body-fixed frame (Seidelmann et al. 2002) using NASA's Navigation and Ancillary Information Facility (NAIF) SPICE tools (Acton 1996; Acton et al. 2018).

\section{RESULTS AND DISCUSSION}

\subsection{Physical characterisation}

We used astrometric observations obtained during the apparition to clearly detect solar radiation pressure acting on $2020 \mathrm{CD}_{3}$ and measure its area-to-mass ratio, $A / m=(0.65 \pm 0.05) \times 10^{-3} \mathrm{~m}^{2} \mathrm{~kg}^{-1}$. This value implies a natural origin for $2020 \mathrm{CD}_{3}$ because it is consistent with $A / m$ for other natural objects in the same size range (Micheli et al. 2012, 2013, 2014; Mommert et al. 2014a,b; Farnocchia et al. 2017) and much lower than typical for artificial objects (Jenniskens et al. 2016).

The derived photometric colors $\left(g^{\prime}-r^{\prime}=0.8 \pm 0.1, r^{\prime}-i^{\prime}=0.15 \pm 0.05\right)$ support $2020 \mathrm{CD}_{3}$ 's natural origin as we do not detect extreme reddening which is associated with artificial objects (Miles 2011). Our broadband photometry suggests that $2020 \mathrm{CD}_{3}$ belongs to the group of silicate asteroids (Fig. 2a), i.e., to the $\mathrm{S}$ or $\mathrm{V}$ complexes in the asteroid taxonomy (DeMeo \& Carry 2013). Based on physical characterization alone, we cannot exclude that $2020 \mathrm{CD}_{3}$ is lunar ejecta, as lunar colors are similar to those of V-type asteroids. The C- and X-complexes, however, can be ruled out.

We also extracted low-precision Gaia G-band photometry (Jordi et al. 2010) from our astrometric observations to derive the photometric phase curve and used it in an independent approach to constrain the spectral classification. 
The observations of $2020 \mathrm{CD}_{3}$ are limited to phase angles $36^{\circ}<\alpha<56^{\circ}$ so that the backscattering region is not covered at all. The poor phase curve coverage does not allow for the photometric data to be fit with the standard $\left(H_{V}, G_{1}, G_{2}\right)$ system in linear brightness space (Muinonen et al. 2010). Instead, we resort to the alternative technique of fitting for $\left(H_{V}, G_{12}\right)$ in nonlinear magnitude space (Penttilä et al. 2016) where $G_{12}$ is forced to stay non-negative and, thus, physically meaningful. The nominal solution, after converting $G_{12}$ to $\left(G_{1}, G_{2}\right)$ and $G$ band to $V$ band assuming $V-G=0.2$, is $\left(H_{V}, G_{1}, G_{2}\right)=\left(31.88_{-0.05}^{+0.03}, 0.0_{-0.0}^{+0.10}, 0.535_{-0.069}^{+0.0}\right)$ (Fig. 2b). We note that the formal uncertainty estimate for $G_{12}$ is meaningless because its nominal value is a result of forcing it to be non-negative and the above uncertainty estimates have been obtained by bootstrapping.

Assuming characteristic slope parameters $\left(G_{1}, G_{2}\right)$ for different asteroid taxonomic types (Shevchenko et al. 2016) and fitting only for $H_{V}$ we find better fits when using slope parameters typical for E, S and $\mathrm{M}$ types than for P, C, and D types (Fig. 2b and Appendix Table 5). Fixing the slope parameters and fitting only for $H_{V}$ results in lower values for the Bayesian Information Criterion than fitting for both $H_{V}$ and $G_{12}$ suggesting that the amount of data is not necessarily sufficient for a meaningful $H_{V}, G_{12}$ fit let alone a full $H_{V}, G_{1}, G_{2}$ fit. The fit is also consistent with slope parameters typical for asteroid (4) Vesta (Gehrels 1967; Shevchenko et al. 2016), the most prominent member of V-type asteroids. These results are in excellent agreement with the photometric colors.

In an alternative, synoptic, approach when fitting the radial component $A_{1}$ to photometry, the fit to the photometric phase curve results in an absolute magnitude $H_{V}=31.9 \pm 0.8$ for $2020 \mathrm{CD}_{3}$. The value is consistent with the purely photometric fit, but the error estimates are more conservative. Assuming the distribution of possible values of the geometric albedo $\left(p_{V}=0.26 \pm 0.08\right.$ for S-types, and $0.34 \pm 0.11$ for V-types, Mainzer et al. 2012) and the phase curve fit for the $H$ magnitude for $\mathrm{S}$ - or V-class asteroids, we obtain a diameter of $1.2_{-0.2}^{+0.4} \mathrm{~m}$, one of the ten smallest NEOs ever found as of 2020 August 10, and amongst the best characterized with colors, rotation period, and AMR. The derived size is consistent with the non-detection of $2020 \mathrm{CD}_{3}$ by the Arecibo radar assuming a non-metallic material composition, excluding an artificial body or an M-type asteroid (Patrick Taylor, personal communication).

Thus, all our evidence suggests that $2020 \mathrm{CD}_{3}$ is of spectral type $\mathrm{S}$ or V. Although little is known of the color distribution of meter-class asteroids, our result is consistent with the observed taxonomic distribution of NEOs with diameters $<200$ meters where S-class objects dominate (Binzel et al. 2019). Furthermore, it is consistent with extrapolations of the asteroid taxonomic and orbital element distribution to small NEOs on Earth-like orbits, the minimoon source population, which suggest that for $H \sim 24.5$, corresponding to S-type asteroids $\sim 40 \mathrm{~m}$ diameter, S-types make up about $40 \%$ of the population (Jedicke et al. 2018b).

The lightcurve of $2020 \mathrm{CD}_{3}$, despite its relatively low signal-to-noise ratio, shows a strong peak at $0.026 \mathrm{~h}$ in a Lomb-Scargle periodogram and a clear minimum in $\chi^{2}$ residuals from Fourier fits to the data at a period $=0.0530 \mathrm{~h}$ (Fig. 3). These reduced $\chi^{2}$ residuals (normalized by the degrees of freedom) were computed for 3rd order Fourier fits across a range of periods from 0.0001 to $2 \mathrm{~h}$ at a step size of $0.0001 \mathrm{~h}$. Second and fourth order Fourier series produced overall higher $\chi^{2}$ values. An approximate 1-sigma error on the period of $0.0011 \mathrm{~h}$ was estimated as the full width at half maximum of the deepest minimum in the $\chi^{2}$ plot. Phasing the data to periods at the limits of this uncertainty range resulted in clear decoherence of the periodic signal. The best-fit period $0.0530 \pm 0.0011 \mathrm{~h}$ is consistent with the LombScargle periodogram. In particular, given the apparent $\sim 0.5$ magnitude peak-to-peak amplitude, the second-order harmonic $(P=0.053 \mathrm{~h})$ is the most probable interpretation of the Lomb-Scargle peak for data obtained at a phase angle of $55^{\circ}$ (Butkiewicz-Bąk et al. 2017). We note that the best-fit rotational period is shorter than the individual integration times of the color photometry. Therefore, the brightness variation due to the rotation of $2020 \mathrm{CD}_{3}$ is averaged out in individual photometric color measurements. Assuming a double-peaked lightcurve, a period of about 3.2 minutes $(\sim 190 \mathrm{~s})$ is a reasonable interpretation, however due to the low signal-to-noise of these data, the period is not strongly constrained. Non-principal axis rotation cannot be ruled out with the available data. The observed rotational period is at least an order of magnitude slower than the predicted mean value from a Maxwellian rotational distribution for meter-sized objects (Bolin et al. 2014). This implies that radar may be better suited for the detection of minimoons than had been previously anticipated, because the radar signal is smeared less by asteroid rotation than suggested by extrapolations of size-rotation-rate models.

The two unknowns in Eq. 1 are the albedo and density but they are constrained by the other measured parameters. Given that our photometric model implies that $2020 \mathrm{CD}_{3}$ is either an $\mathrm{S}$ or $\mathrm{V}$ type asteroid, we generated synthetic albedos for 10000 of each type according the type-specific albedo distributions of Mainzer et al. (2012). Similarly, we generated the same number of random $A_{1}$ values using a normal distribution with a mean and width given by the central value and uncertainty on our measured $A_{1}=(3.1 \pm 0.2) \times 10^{-9} \mathrm{~m} \mathrm{~s}^{-2}$. The pairs of synthetic albedo- $A_{1}$ values 
a)

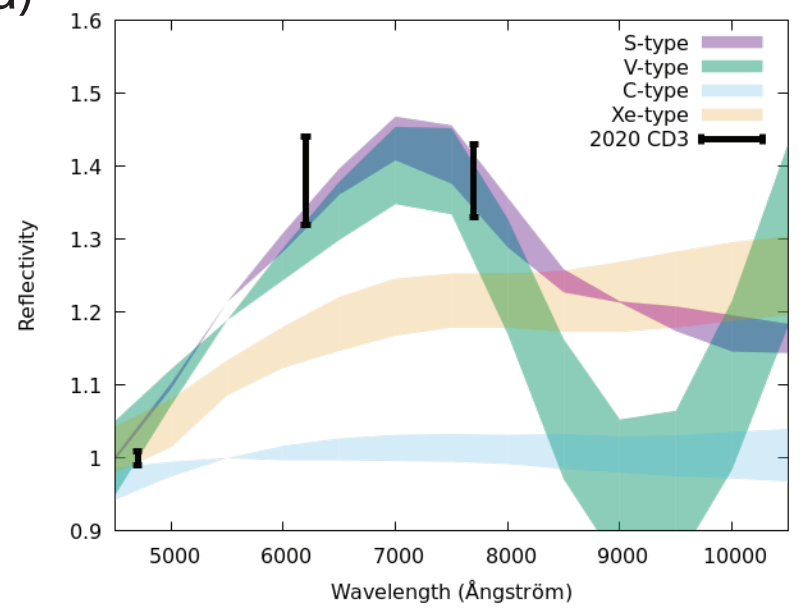

c)

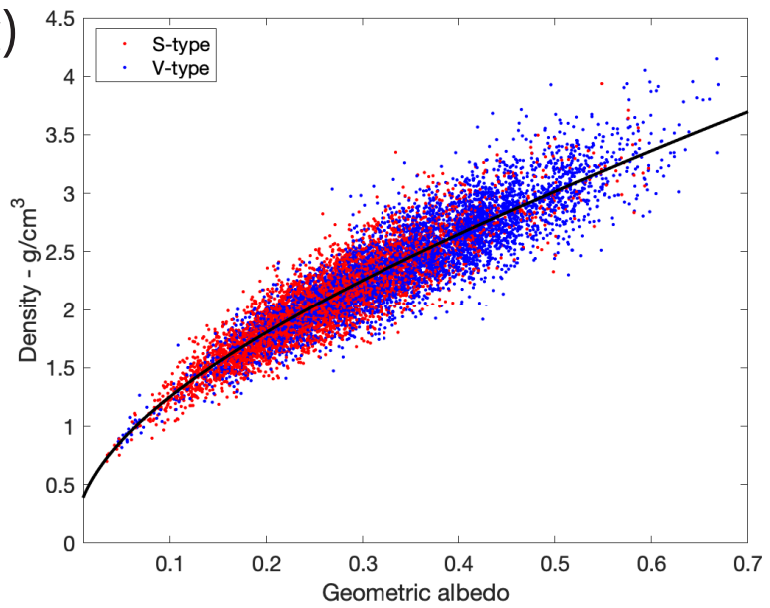

b)

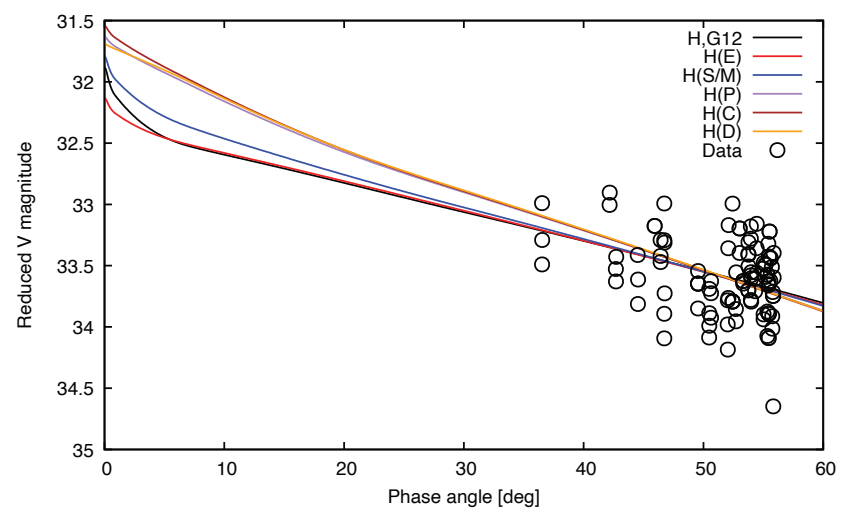

d)

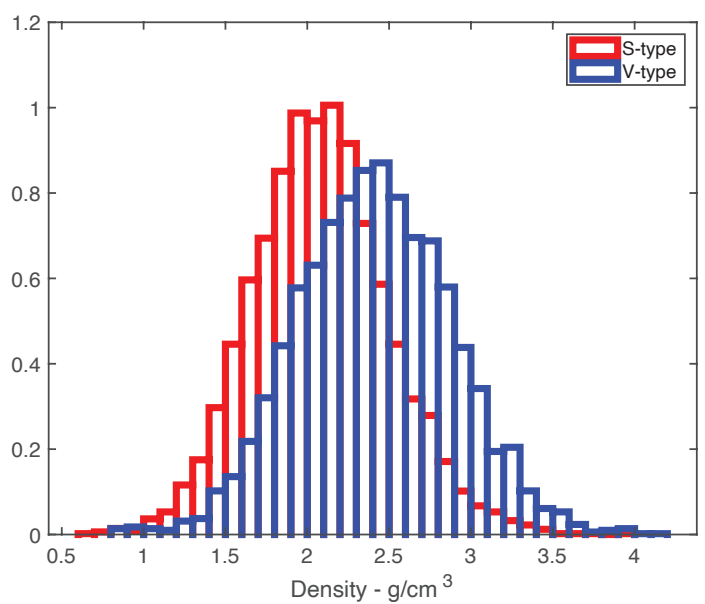

Figure 2. Physical characterisation of $2020 \mathrm{CD}_{3}$. (a) Comparison of the color indices of $2020 \mathrm{CD}_{3}$ obtained with GMOS/Gemini North (black) to the reflectance spectra of the main asteroid taxonomic complexes (DeMeo et al. 2009). Spectral types $\mathrm{C}$ (azure) and Xe (nude, the reddest member of the X-complex) can be ruled out, leaving types $\mathrm{S}$ (violet) and V (turquoise) as plausible choices. (b) Photometric phasecurve and constrained photometric fits for $2020 \mathrm{CD}_{3} . H, G_{12}$ is the nonlinear, constrained two-parameter fit, and $H(\ldots)$ refer to one-parameter fits were the slope parameters have been fixed to typical values for different spectral types (E,S/M,P,C,D). (c) Scatter plot of albedo and bulk density. Red and blue dots correspond to our Monte Carlo distributions for the $\mathrm{S}$ and $\mathrm{V}$ taxonomic classes, respectively. The black curve corresponds to the best-fit to the density as a function of the albedo. (d) Monte Carlo distribution of the density of $2020 \mathrm{CD}_{3}$ for the $\mathrm{S}$ (red) and $\mathrm{V}$ (blue) taxonomic classes.

were then used to calculate the object's density (Fig. 2c-d). For the S-type assumption we find $\rho=2.1 \pm 0.4 \mathrm{~g} \mathrm{~cm}^{-3}$ whereas for the V-type assumption $\rho=2.4 \pm 0.5 \mathrm{~g} \mathrm{~cm}^{-3}$. In both cases, the inferred density is consistent with typical asteroid densities (Carry 2012). We note that the possible effect of the Yarkovsky force can potentially increase the estimated density values by 10-20\% (Chesley et al. 2014) so that our density estimates represent the lower bound of values. However, this does not have a major impact on the interpretation of the results.

Thus, our physical characterization of $2020 \mathrm{CD}_{3}$ indicates that it is a silicate body, perhaps a free-floating analogue of what appear to be monolithic boulders found on the surface of larger asteroids such as (25143) Itokawa, the S-type asteroid investigated in-situ by the Hayabusa spacecraft (Saito et al. 2006). Alternatively, it could be a small rubblepile aggregate more like the $2008 \mathrm{TC}_{3}$ (e.g. Jenniskens et al. 2009). While the internal structure of meter-scale asteroids 
2020 CD3; LDT/LMI; 2020-02-27
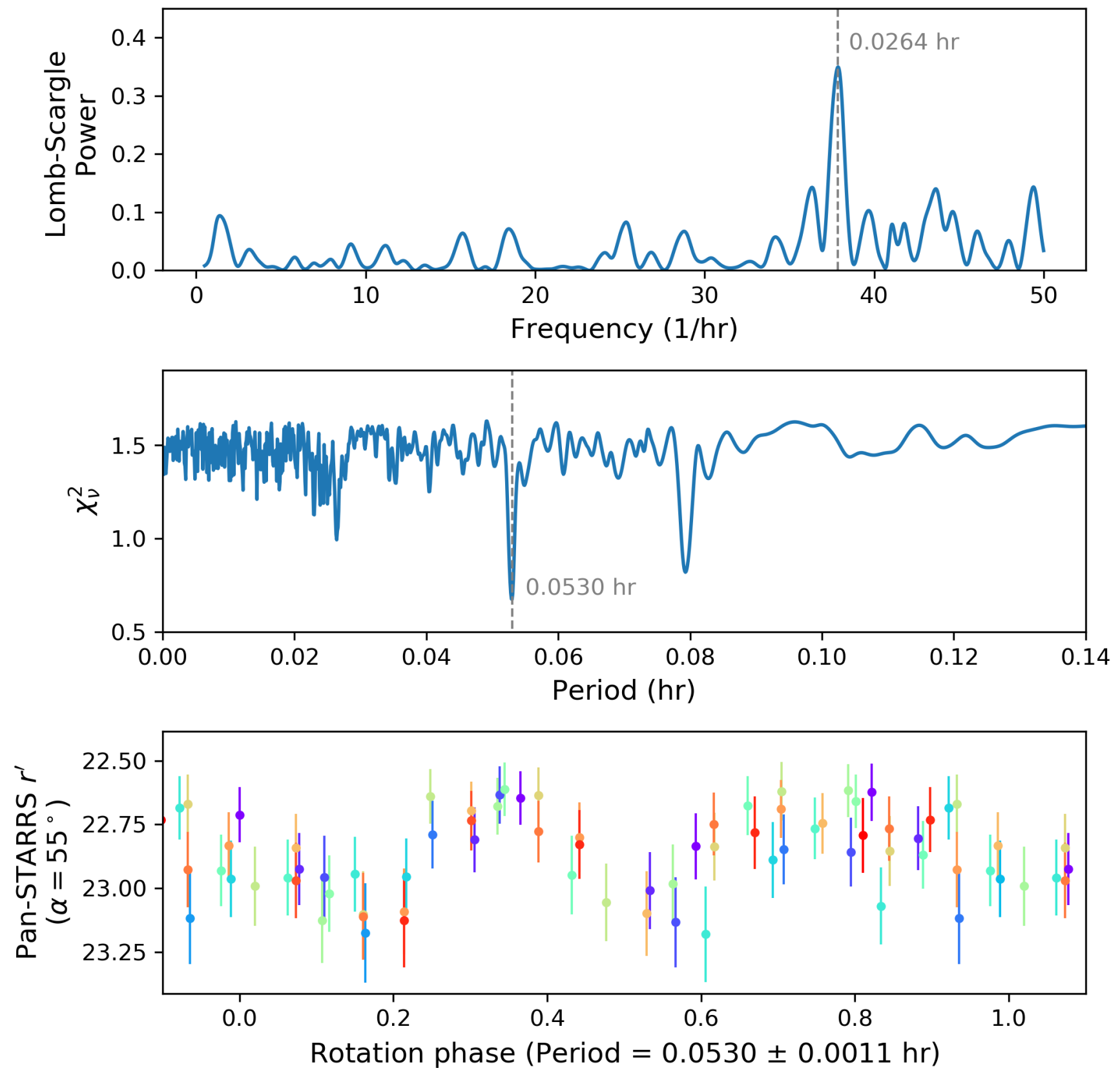

Figure 3. Lightcurve of $2020 \mathbf{C D}_{3}$. Top panel: the Lomb-Scargle periodogram for the rotation period of $2020 \mathrm{CD}_{3}$. With $30 \mathrm{~s}$ exposures taken over a span of 60 minutes, the range of periodogram frequencies is limited from 70 seconds to 2 hours. The peak in the periodogram is at $0.0264 \mathrm{~h}$. Middle panel: reduced $\chi^{2}$ residuals from lightcurve curve fits using a $3 r d$ order Fourier series. The best fit at 0.0530 hours corresponds to the full rotational period. Bottom panel: LDT lightcurve photometry of $2020 \mathrm{CD}_{3}$ calibrated to the Pan-STARRS $r_{\mathrm{P} 1}$ filter. The 1-hour sequence of data has been phase folded to the best fit period of $0.0530 \mathrm{~h}$ (3.2 minutes). The color scale represents the ordering of the measurements from the beginning of the sequence in blue, to the end in red. 
is currently unknown we expect that favourable appearances of small NEOs and future minimoons will provide more opportunities for detailed characterization of these small asteroids.

\subsection{Orbital evolution}

Integrating $2020 \mathrm{CD}_{3}$ 's trajectory into the past indicates that it was bound to the Earth and it's orbit was deterministic after a close approach to the Moon on 2017 September 15 (Figure 4a). Prior to this encounter there are three possible behaviors: 1) $97.3 \%$ escape the Earth-Moon system, corresponding to a scenario in which this encounter is responsible for the capture of $2020 \mathrm{CD}_{3}$ by the Earth-Moon system; 2) $1.4 \%$ intersect the Moon's surface, which corresponds to the hypothesis that $2020 \mathrm{CD}_{3}$ is lunar ejecta; 3) $1.3 \%$ remain in Earth orbit (potentially for more than 10 years). Therefore, we conclude that $2020 \mathrm{CD}_{3}$ was in orbit around Earth since at least 2017 September 15. Since then, it completed 11 orbits around the Earth with intervals between successive perigees of 70 to 90 days. Its minimum geocentric distance was between 12900 and $13400 \mathrm{~km}$ on 2019 April 4 and it escaped the Earth's Hill sphere $(\sim 0.01$ au) on 2020 March 7 after a final perigee on 2020 February 13 at a geocentric distance of about $47000 \mathrm{~km}$. Oddly, it passed its last perigee just two days before its discovery. $2020 \mathrm{CD}_{3}$ 's Earth-like orbit means it has a long synodic orbital period so it will not approach Earth again until March 2044 at about 10 lunar distances, well outside Earth's Hill sphere.

The capture duration of $2020 \mathrm{CD}_{3}$ of at least 2.7 years (Fig. 4b) may seem exceptionally long considering that orbital simulations suggest that the average capture duration of minimoons is about nine months (Fedorets et al. 2017). However, there is an inverse correlation between the average capture duration and the minimum lunacentric distance when the encounter distance is less than $30000 \mathrm{~km}$ (Fig. 4c). In such cases, minimoons may become captured for years or even decades. Although only $\sim 2 \%$ of minimoons have capture durations greater than three years those objects' total capture duration time is $23 \%$ of the cumulative capture duration time of all simulated minimoons. Based on the close encounter of $2020 \mathrm{CD}_{3}$ with the Moon, it is not surprising that $2020 \mathrm{CD}_{3}$ undergoes a longer geocentric capture than an average minimoon. The distribution of possible capture durations of $2020 \mathrm{CD}_{3}$ is thus in agreement with theoretical predictions (Fig. 4b).

We argue that a lunar origin for $2020 \mathrm{CD}_{3}$ is highly unlikely (see Subsect. 4.3) and therefore assume that the object originated in the main asteroid belt. Based on its pre-capture heliocentric orbit it has a (72 \pm 1$) \%$ probability of having been ejected by the $\nu_{6}$ secular resonance with, primarily, Saturn (Granvik et al. 2018). A provenance in the inner main belt would also favor its identification in the S-type taxonomy since S-types dominate that region of the belt. There is a $(28 \pm 1) \%$ for it having originated in the Hungaria region and a negligible $(0.5 \pm 0.03) \%$ probability that it was ejected from the outer region of the main belt by the 3:1 mean-motion resonance with Jupiter. The reported uncertainties on the probabilities are the standard error on the mean across several discrete cells in the Granvik et al. (2018) NEO population model. An inner belt source for $2020 \mathrm{CD}_{3}$ is in agreement with a silicate-rich asteroid composition which is dominant in that region (DeMeo \& Carry 2014).

\subsection{Exploring the lunar ejecta hypothesis}

There is a possibility that $2020 \mathrm{CD}_{3}$ could have been spall ejected by a recent lunar impact event (Subsect. 4.2) and we assess the likelihood of this scenario by examining the contemporary production rate of small craters on the Moon.

The largest crater to form annually on the Moon is approximately $50 \mathrm{~m}$ in diameter based on a survey of fresh impact craters identified on the Moon using "before" and "after" images from the Lunar Reconnaissance Orbiter (LRO) Narrow Angle Camera (NAC) (Speyerer et al. 2016). Accordingly, if $2020 \mathrm{CD}_{3}$ was launched from the lunar surface on 2017 September 15 a crater of this scale would need to be capable of launching a meter-sized minimoon off the Moon and onto the trajectory described above.

An asteroid striking the Moon creates a crater approximately 20 times its own size (Melosh 1989) so a $2.5 \mathrm{~m}$ diameter projectile can make a $50 \mathrm{~m}$ diameter crater. Hirase et al. (2004) investigated the relationship of ejecta velocity relative to the ejecta-to-impactor diameter ratio in laboratory experiments, an analysis of secondary craters produced by lunar and Martian craters, and ejecta from the asteroid (4) Vesta that make up the Vesta family (often called Vestoids). At an ejecta/impactor diameter ratio of $\sim 0.4$, corresponding to the ejection of a $1 \mathrm{~m}$ diameter minimoon by a $2.5 \mathrm{~m}$ diameter projectile, the typical ejection speed are a few tens of $\mathrm{m} \mathrm{s}^{-1}$ and certainly $<100 \mathrm{~m} \mathrm{~s}^{-1}$ - much smaller than lunar escape velocity $\left(\sim 2400 \mathrm{~m} \mathrm{~s}^{-1}\right)$. Indeed, the results of Hirase et al. (2004)'s analysis suggest that launching a $1 \mathrm{~m}$ diameter minimoon off the lunar surface requires the impact of a km-scale asteroid, an unlikely event that surely would have been noticed on or soon after 2017 September 15. Furthermore, the population of NEOs is $\gtrsim 90 \%$ known 


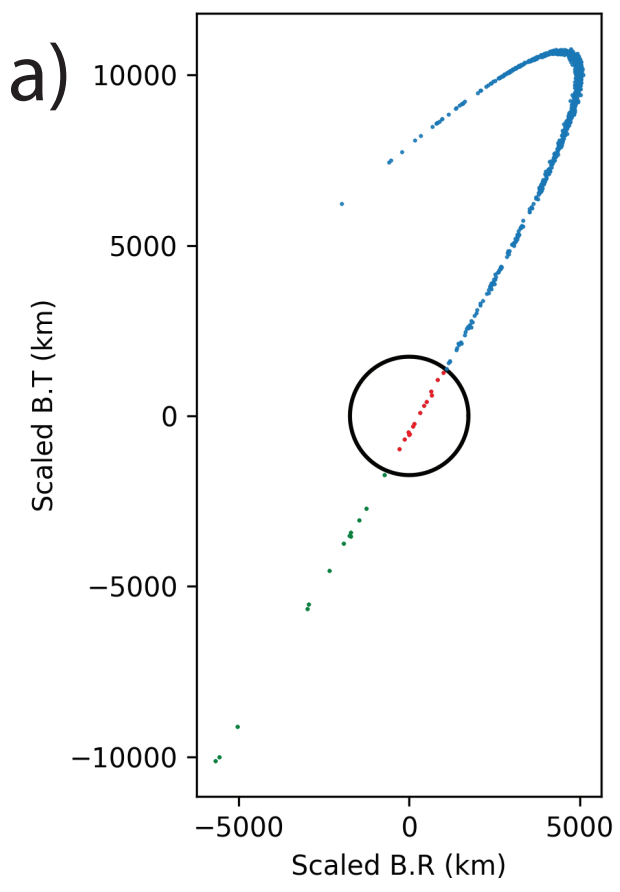

b)
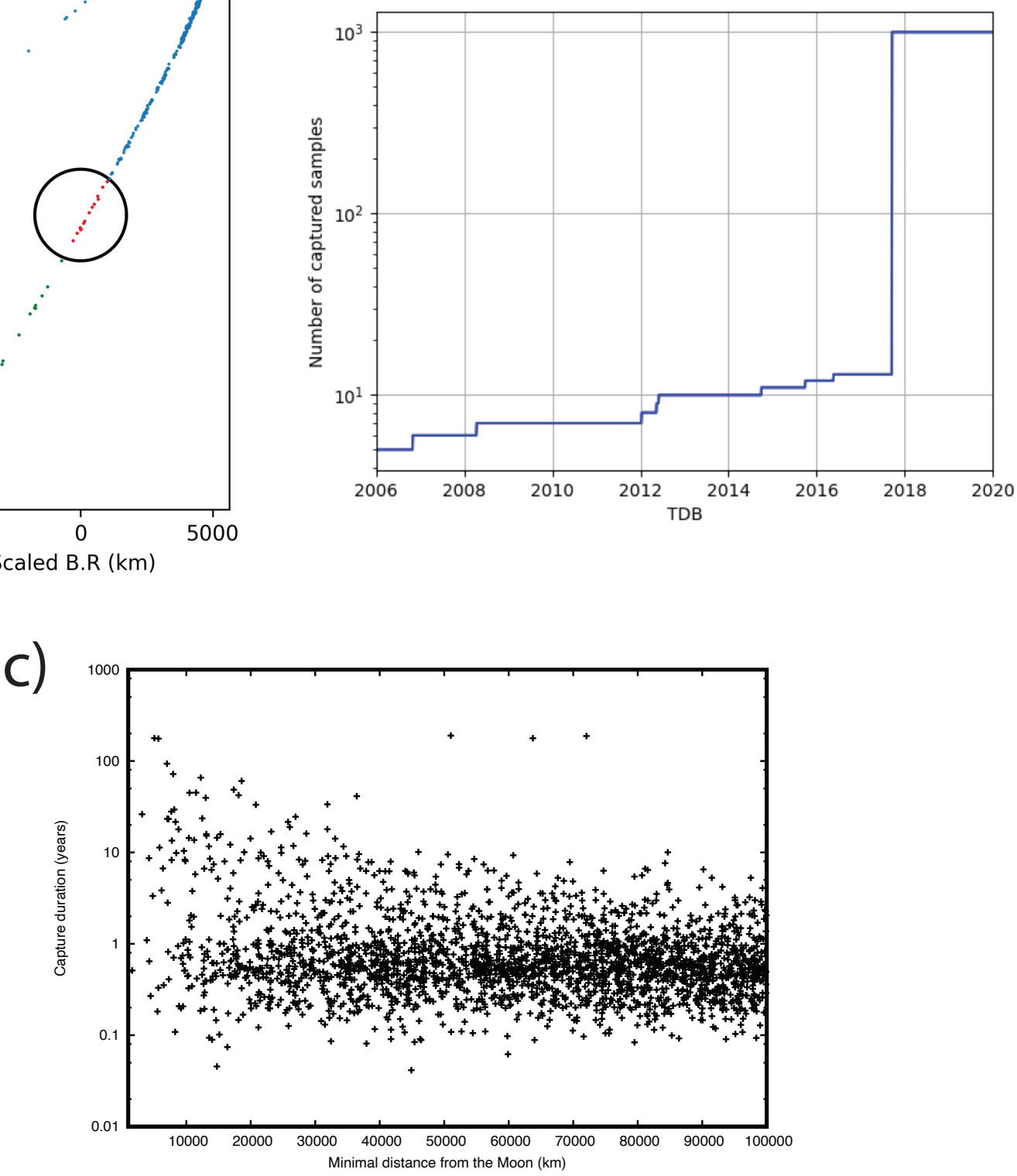

Figure 4. Orbital evolution of $2020 \mathbf{C D}_{3}$. (a) Monte Carlo samples of the $2020 \mathrm{CD}_{3}$ trajectory mapped onto the outbound scaled B-plane (Farnocchia et al. 2019) of the Moon (black circle) on 2017 September 15. On that date there was a close encounter between $2020 \mathrm{CD}_{3}$ and the Moon which results in a trivergence of orbital solutions when going further back in time: (blue) samples captured on a geocentric orbit during this encounter, (red) samples originating from the Moon, and (green) samples remaining in an orbit around Earth prior to the encounter. (b) The cumulative number of Monte Carlo samples bound to the Earth-Moon system as a function of time when integrating backwards from 2020. (c) The capture duration of minimoons as a function of their minimum lunacentric distance (using data from Fedorets et al. 2017). A close encounter between a minimoon and the Moon typically increases the capture duration. 
at this time and no impacts were predicted on that date. Accordingly, we reject a lunar ejecta origin for $2020 \mathrm{CD}_{3}$. In summary, while NEO-based models (Granvik et al. 2012; Fedorets et al. 2017) indicate that an annual capture of a meter-sized asteroid is likely, the production of similar-sized lunar ejecta at the same rate can be ruled out. Hence, minimoon capture from the NEO population is a dominating mechanism for maintaining the minimoon steady-state population.

An additional blow to the lunar origin hypothesis for $2020 \mathrm{CD}_{3}$ comes from lunar meteorites that were blasted off the Moon in the past. Warren (1994), whose analysis builds on the work of Melosh (1985), argues that most lunar meteorites came from lunar craters that were hundreds of meters to several $\mathrm{km}$ in diameter and that the meteoroid precursor bodies to the meteorites were $2-10 \mathrm{~cm}$ in diameter prior to entering Earth's atmosphere. Lunar meteorite cosmic ray exposure ages indicate that only about half took less than 100000 years to get to Earth (Warren 1994). Given that minimoon orbital lifetimes are typically on the order of a year it implies that those meteoroids spent most of their time on heliocentric orbits before being delivered back to Earth, not in the Earth-Moon system. Taken together, it suggests that it is difficult for small craters to launch sizeable bodies off the Moon; if small impact events could do so, we might expect very young lunar meteorites to dominate the fall and find record on Earth.

We emphasize that the impact capable of producing an ejecta of the size of $2020 \mathrm{CD}_{3}$ would have been very bright. Moreover, the distribution of the subset of sample orbits originating from the Moon point the majority of them to the part of the dark size of the Moon facing towards Earth, providing optimal observing conditions (Appendix Fig. 6). No major impacts have been reported, including the NELIOTA telescope (Xilouris et al. 2018), the NASA lunar impact monitoring (William Cooke, private communication). Moreover, no reports of a new km-sized craters on the Moon have been announced.

In summary, we consider the lunar origin of $2020 \mathrm{CD}_{3}$ to be extremely unlikely.

\subsection{Detectability of $2020 C D_{3}$}

The discovery of $2020 \mathrm{CD}_{3}$ occurred at the last window of opportunity (Fig. 5). However, simulations by Fedorets et al. (2020), and the fact that $2006 \mathrm{RH}_{120}$ was discovered only three months into its captured time period of one year, suggest that the last-minute discovery of $2020 \mathrm{CD}_{3}$ is not a typical situation. During the undisputed capture period of 2.7 years there were six distinct intervals during which $2020 \mathrm{CD}_{3}$ was brighter than the discovery observatory's (CSS's Mt. Lemmon) limiting magnitude (Fig. 5). It even briefly reached $V<16$ when it approached to within about $20000 \mathrm{~km}$, below the orbits of geosynchronous satellites. The problem is that during the detectability windows, when it was bright and close to Earth, it also had a high apparent rate of motion so that it would have left a trailed image on the detector, spreading out the light from the object and reducing the per pixel signal-to-noise ratio (SNR) to a level below the system's detection threshold. Taking these trailing losses into account, there were only three 2-hour time segments during the entire 2.7 years in which $2020 \mathrm{CD}_{3}$ was detectable by the Mt. Lemmon telescope, corresponding to $\sim 0.03 \%$ of the time under the best of circumstances. A similar analysis for the Pan-STARRS1 telescope (Chambers et al. 2016) finds that there were only four short time periods during which it could have detected $2020 \mathrm{CD}_{3}$. PanSTARRS1 reaches a fainter limiting magnitude than Mt. Lemmon due to its larger aperture and better seeing statistics but its smaller pixels makes it less sensitive to fast-moving objects like minimoons.

\subsection{Minimoon population}

We expect there to exist a much larger but undiscovered population of minimoons that are similar or smaller in size to $2020 \mathrm{CD}_{3}$ (Fedorets et al. 2017; Granvik et al. 2012) - they are just difficult to detect due to their faintness, rate of motion, and infrequent windows of observational opportunity (Fig. 5). Estimating the minimoon populations size-frequency distribution by debiasing the discovered population of two objects is essentially impossible given that they are so difficult to detect and were at the limit of the system's detection capability. In addition to the two minimoons discovered by CSS, observations of meteors created by meteoroids that had a high probability of being geocentric prior to entering the atmosphere (Clark et al. 2016; Shober et al. 2019) support the existence of a minimoon population. These meteor observations are also difficult to convert into a minimoon population estimate because a meteor's apparent brightness, in both the optical and radar, is dominated by the meteoroids diameter and its speed. Since meteors generated by minimoons have the lowest possible meteor speed, essentially equal to Earth's escape speed, they are the faintest possible meteors. Thus, in order for them to be bright enough to be detected they must be large and therefore rare.

To quantify the detection frequency of minimoons we apply Bolin et al. (2014)'s modelling of the performance of Pan-STARRS1 survey (PS1; Chambers et al. 2016) to the CSS's Mt. Lemmon observatory that discovered both of 


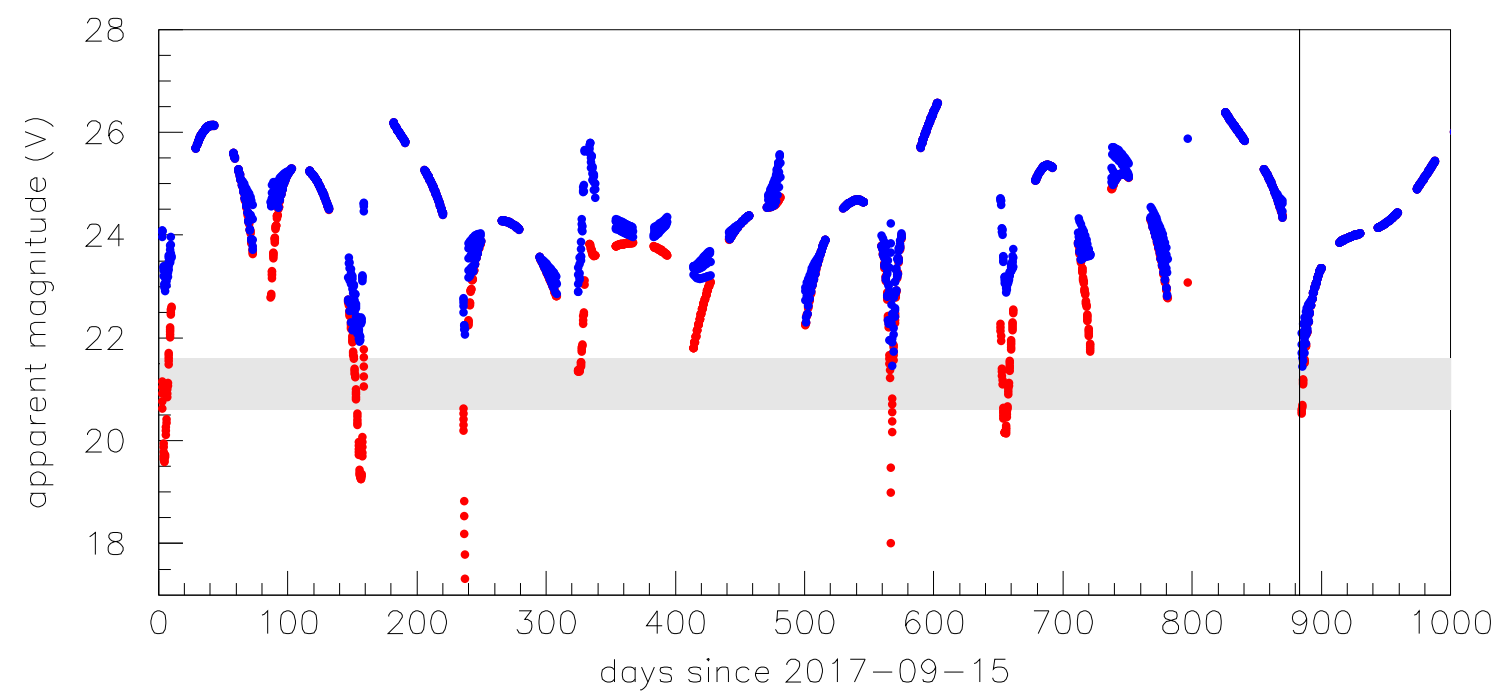

Figure 5. Detectability windows for $2020 \mathbf{C D}_{3}$. The red points represent $2020 \mathrm{CD}_{3}$ 's V-band apparent magnitude every 2 hours over a period of about 2.7 years beginning on the date of its close approach to the Moon on 2017 September 15 . The blue points represent $2020 \mathrm{CD}_{3}$ 's V-band apparent magnitude after accounting for trailing losses specific to the discovery observatory. To mimic CSS's observing strategy, the points are only shown when $2020 \mathrm{CD}_{3}$ is more than $60^{\circ}$ from the Sun, more than $45^{\circ}$ from the Moon when it is $<50 \%$ illuminated, and from $4 \mathrm{~h}$ to $12 \mathrm{~h}$ UTC. The vertical line is on the date on which $2020 \mathrm{CD}_{3}$ was discovered (2020 February 15). The horizontal gray band represents \pm 0.5 magnitudes centered on the average limiting magnitude of the CSS Mt. Lemmon telescope. Thus, the only time when that telescope could detect $2020 \mathrm{CD}_{3}$ is when blue dots appear in or below the gray band.

the telescopically identified minimoons. The application is appropriate because the two observatories have roughly similar capabilities, especially considering all the difficulties involved in modeling the detection of faint, fast-moving minimoons, and the statistics of just two objects. The modelled PS1 survey has a peak probability of detecting minimoons at $H_{V}=31.5 \pm 1.5$ so Mt. Lemmon's discovery of $2020 \mathrm{CD}_{3}$ with $H_{V}=31.9 \pm 0.8$ is not surprising. Furthermore, Bolin et al. (2014) estimated that PS1 (and therefore Mt. Lemmon) could detect about 0.01 minimoons per lunation or about one every $\sim 8.1$ years as compared to the $\sim 14$ year interval since CSS's discovery of $2006 \mathrm{RH}_{120}$. We think the $\sim 2 \times$ discrepancy in the time interval is not significant given that 1) Bolin et al. (2014) used the earlier and larger minimoon size-frequency distribution of Granvik et al. (2012) compared to the revised distribution of Fedorets et al. (2017) and because 2) it is intrinsically difficult to model discovery rates at the limits of detectability in both flux and rate of motion (see Fig. 5). Moreover, assuming Poisson-like discovery statistics, and that the CSS Mt. 
Lemmon survey has been in operation at roughly the same capability level for 20 years, over the same period there is a $\sim 68 \%$ probability of discovering $\leq 2$ minimoons. Therefore, the discovery of $2020 \mathrm{CD}_{3} 14$ years after the discovery of $2006 \mathrm{RH}_{120}$, a minimoon with $H_{V}=29.9 \pm 0.3$, is in line with the capture frequency of minimoons predicted by existing population models, and consistent with their predicted discovery rate (Bolin et al. 2014).

An additional complication in debiasing the minimoon population identified in asteroid surveys is the difficulty of identifying rare natural objects among numerous artificial ones (Jedicke et al. 2018a). As sky surveys have become more powerful and efficient at identifying faint and trailed objects they have been detecting ever more artificial geocentric objects, often on minimoon-like orbits. Distinguishing both $2020 \mathrm{CD}_{3}$ and $2006 \mathrm{RH}_{120}$ from artificial objects upon their discovery was initially inadvertently affected by human biases that objects on geocentric orbits are artificial and correcting the observation statistics for this bias will be difficult.

\section{CONCLUSIONS}

We provide an in-depth study of the orbital and physical characteristics of Earth's second discovered minimoon, $2020 \mathrm{CD}_{3}$. The combination of its area-to-mass ratio derived from its solar radiation pressure signature, its reflectance spectrum that is consistent with silicate asteroids, and its non-detection by radar provides evidence that $2020 \mathrm{CD}_{3}$ is a natural object. Its derived geometric albedo - bulk density distributions are consistent with values typical of silicate asteroids. $2020 \mathrm{CD}_{3}$ could be a free-floating silicate analogue of boulders discovered on surfaces of larger asteroids.

High precision astrometry enabled by the Gaia mission permits the measurement of ground-based astrometry of asteroids to a level of $0.05^{\prime \prime}$ in the best cases. This, in turn, provides better, faster constraints on the solar radiation pressure signature for small asteroids. For the best possible results, special attention needs to be paid to 1) using accurate and precise geographical coordinates for ground-based observatories and 2) time-keeping.

The geocentric orbital evolution of $2020 \mathrm{CD}_{3}$ includes a close dynamical interaction with the Moon on 2017 September 15. It was bound on a geocentric orbit for at least 2.7 years, which makes it an exceptionally long capture compared to typical capture duration results from simulations. However, a long duration is actually typical for minimoons that experience close approaches to the Moon. Some orbital trajectories suggest a lunar ejecta origin of $2020 \mathrm{CD}_{3}$, but we showed that this possibility is unlikely.

The discovery of $2020 \mathrm{CD}_{3}$ is in line with the most recent theoretical predictions for the steady-state population of minimoons (Fedorets et al. 2017), supporting the prediction for an increased discovery rate of minimoons (Fedorets et al. 2020) with the upcoming Vera C. Rubin Observatory's Legacy Survey of Space and Time (LSST; Ivezić et al. 2019). More discoveries of minimoons are also anticipated through improvements to the ongoing NEO surveys such as CSS (Bolin et al. 2014) but trailing losses remain a major factor affecting the discovery of minimoons. A rapid determination of the nature of temporarily-captured objects after their discovery remains a challenge which is expected to become even more pressing with the anticipated increase in their discovery rate with LSST.

The discovery and characterization of $2020 \mathrm{CD}_{3}$ paves the way for the observational study of minimoons as a population instead of a curiosity with serendipitous discoveries, as well as for identifying candidate targets for costeffective space missions to these unexplored objects at the asteroid-meteoroid boundary (Granvik et al. 2013; Jedicke et al. 2018a).

\section{ACKNOWLEDGMENTS}

The authors thank Bryce Bolin, William Cooke, Alan Fitzsimmons, Tomáš Kohout, Antti Penttilä, Patrick Taylor and Anne Virkki for helpful discussions, the anonymous referee for his/her insightful comments, and Daniel Hestroffer for his hospitality during the research visit of G.F. to the Paris Observatory.

Based on observations made with the Nordic Optical Telescope, operated by the Nordic Optical Telescope Scientific Association at the Observatorio del Roque de los Muchachos, La Palma, Spain, of the Instituto de Astrofisica de Canarias. The data presented here were obtained in part with ALFOSC, which is provided by the Instituto de Astrofisica de Andalucia (IAA) under a joint agreement with the University of Copenhagen and NOTSA.

Based on observations obtained with MegaPrime/MegaCam, a joint project of CFHT and CEA/DAPNIA, at the Canada-France-Hawaii Telescope (CFHT) which is operated by the National Research Council (NRC) of Canada, the Institut National des Sciences de l'Univers of the Centre National de la Recherche Scientifique (CNRS) of France, and the University of Hawaii. The observations at the CFHT were performed with care and respect from the summit of Maunakea which is a significant cultural and historic site. 
The authors acknowledge the sacred nature of Maunakea, and appreciate the opportunity to obtain observations from the mountain. This work is partly based on observations obtained at the international Gemini Observatory, a program of NSF's OIR Lab, which is managed by the Association of Universities for Research in Astronomy (AURA) under a cooperative agreement with the National Science Foundation. on behalf of the Gemini Observatory partnership: the National Science Foundation (United States), National Research Council (Canada), Agencia Nacional de Investigación y Desarrollo (Chile), Ministerio de Ciencia, Tecnología e Innovación (Argentina), Ministério da Ciência, Tecnologia, Inovações e Comunicações (Brazil), and Korea Astronomy and Space Science Institute (Republic of Korea). The observations were obtained as part of Gemini Director's Discretionary Program GN-2020A-DD-107. The GMOSN observations were acquired through the Gemini Observatory Archive at NSF's NOIRLab and processed using DRAGONS (Data Reduction for Astronomy from Gemini Observatory North and South).

This work was enabled by observations obtained with the University of Hawai'i's 2.2-meter telescope and the Gemini North telescope, and the Canada-France-Hawaii telescope, all located within the Maunakea Science Reserve and adjacent to the summit of Maunakea. We are grateful for the privilege of observing the Universe from a place that is unique in both its astronomical quality and its cultural significance.

These results made use of the $4.3 \mathrm{~m}$ Lowell Discovery Telescope at Lowell Observatory. Lowell is a private, nonprofit institution dedicated to astrophysical research and public appreciation of astronomy and operates the LDT in partnership with Boston University, the University of Maryland, the University of Toledo, Northern Arizona University and Yale University. The Large Monolithic Imager was built by Lowell Observatory using funds provided by the National Science Foundation (AST-1005313). Part of the LDT observations have been obtained thanks to the NASA SSO-NEOO grant number 80NSSC19K1586. Some of the LDT observations were obtained by the University of Maryland observing team, consisting of L. M. Feaga, Q.-Z. Ye, J. M. Bauer, T. L. Farnham, C. E. Holt, M. S. P. Kelley, J. M. Sunshine, and M. M. Knight.

This work has made use of data from the European Space Agency (ESA) mission Gaia (https://www.cosmos.esa. int/gaia), processed by the Gaia Data Processing and Analysis Consortium (DPAC, https://www.cosmos.esa.int/ web/gaia/dpac/consortium). Funding for the DPAC has been provided by national institutions, in particular the institutions participating in the Gaia Multilateral Agreement.

Based on the observations obtained with the Chinese Near-Earth Object Survey Telescope (CNEOST). We thank Bin Li for providing the exposure catalog.

Based on observations obtained with the Samuel Oschin 48-inch Telescope at the Palomar Observatory as part of the Zwicky Transient Facility project. ZTF is supported by the National Science Foundation under Grant No. AST1440341 and a collaboration including Caltech, IPAC, the Weizmann Institute for Science, the Oskar Klein Center at Stockholm University, the University of Maryland, the University of Washington, Deutsches Elektronen-Synchrotron and Humboldt University, Los Alamos National Laboratories, the TANGO Consortium of Taiwan, the University of Wisconsin at Milwaukee, and Lawrence Berkeley National Laboratories. Operations are conducted by COO, IPAC, and UW.

The Catalina Sky Survey is funded since 1998 by the National Aeronautics and Space Administration's Near Earth Object Observations program, currently under Grant No. 80NSSC18K1130.

The Pan-STARRS1 Surveys (PS1) have been made possible through contributions by the Institute for Astronomy, the University of Hawaii, the Pan-STARRS Project Office, the Max-Planck Society and its participating institutes, the Max Planck Institute for Astronomy, Heidelberg and the Max Planck Institute for Extraterrestrial Physics, Garching, The Johns Hopkins University, Durham University, the University of Edinburgh, the Queen's University Belfast, the Harvard-Smithsonian Center for Astrophysics, the Las Cumbres Observatory Global Telescope Network Incorporated, the National Central University of Taiwan, the Space Telescope Science Institute, and the National Aeronautics and Space Administration under Grant No. NNX08AR22G issued through the Planetary Science Division of the NASA Science Mission Directorate, the National Science Foundation Grant No. AST-1238877, the University of Maryland, Eötvös Loránd University (ELTE), and the Los Alamos National Laboratory.

M.M. would like to thank Detlef Koschny, Luca Conversi and Erwin Schwab for their support with the CAHA Schmidt observations.

G.F. was supported by STFC Grant ST/P000304/1. M.G. was partly supported by the Academy of Finland. Support for M.D. and N.M. was provided by NASA NEOO grant NNX17AH06G in support of the Mission Accessible NearEarth Object Survey (MANOS). W.B. and R.J. were supported in part by NASA's Near Earth Object Observations program (Grant Number 80NSSC17K0153). L.B. acknowledges funding from the Science Technology Funding Council 
(STFC) Grant Code ST/T506369/1. T.S. is supported by Gemini Observatory through a Gemini Science Fellowship. Part of this research was conducted at the Jet Propulsion Laboratory, California Institute of Technology, under a contract with NASA.

This work made use of NASA's Astrophysics Data System Bibliographic Services and the data and services provided by the International Astronomical Union's Minor Planet Center. (https://minorplanetcenter.net/data).

\section{AUTHOR CONTRIBUTIONS}

G.F., M.M., R.J., S.N, D.Fa., M.G., N.M., M.S., R.We., K.W., E.C., Q.Y., W.B. wrote the paper. K.W. and T.P. discovered the object. G.F and M.M. acquired the NOT data, supported by A.D. R.We. and R.Wa. acquired the CFHT data. N.M., Q.Y., M.D. and M.M. acquired the LDT data. D.Fö. acquired the UH88 data. G.F., M.S., L.B., M.M., M.G. wrote the Gemini North DD proposal. M.S., G.F., T.S., D.M.F., J.R., A.S. acquired the Gemini North data. M.M., R. We., Q.Y. searched for precovery images. M.M. and D.Fa. computed the area-to-mass ratio. N.M. derived the rotational period. D.Fa., M.M., M.G., R.J., G.F. physically characterised the object. S.N., D.Fa. and M.M. calculated the orbit. W.B. and R.J. investigated the lunar ejecta hypothesis. M.G. estimated the source region of the object. R.J., E.C., R.We. and M.M. investigated the detectability of the object. R.J., G.F., M.M., M.G., D.Fa. interpreted the results.

Software: DAOPHOT (Stetson 1987), IRAF (Tody 1986, 1993), NASA NAIF SPICE tools (Acton 1996, Acton et al. 2018), Sextractor (Bertin \& Arnouts 1996), DRAGONS (AURA Gemini Observatory-Science User Support Department 2018), Photometry Pipeline (Mommert 2017).

\section{REFERENCES}

Acton, C., Bachman, N., Semenov, B., \& Wright, E. 2018, Planetary and Space Science, 150, 9 , doi: https://doi.org/10.1016/j.pss.2017.02.013

Acton, C. H. 1996, Planet. Space Sci., 44, 65, doi: 10.1016/0032-0633(95)00107-7

Alam, S., Albareti, F. D., Allende Prieto, C., et al. 2015, ApJS, 219, 12, doi: 10.1088/0067-0049/219/1/12

AURA Gemini Observatory-Science User Support Department. 2018, DRAGONS: Gemini Observatory data reduction platform. http://ascl.net/1811.002

Bellm, E. C., Kulkarni, S. R., Graham, M. J., et al. 2019, PASP, 131, 018002, doi: 10.1088/1538-3873/aaecbe

Bertin, E., \& Arnouts, S. 1996, A\&AS, 117, 393, doi: $10.1051 /$ aas:1996164

Binzel, R., DeMeo, F., Turtelboom, E., et al. 2019, Icarus, 324, 41 , doi: https://doi.org/10.1016/j.icarus.2018.12.035

Bolin, B., Jedicke, R., Granvik, M., et al. 2014, Icarus, 241, 280

Bressi, T. H., Hergenrother, C. W., Christensen, E. J., et al. 2008, Minor Planet Electronic Circulars, 2008-D12

Brown, P., Spalding, R. E., ReVelle, D. O., Tagliaferri, E., \& Worden, S. 2002, Nature, 420, 294

Brown, P. G., Assink, J. D., Astiz, L., et al. 2013, Nature, 503, 238 , doi: 10.1038/nature12741

Butkiewicz-Bąk, M., Kwiatkowski, T., Bartczak, P., Dudziński, G., \& Marciniak, A. 2017, Monthly Notices of the Royal Astronomical Society, 470, 1314
Carry, B. 2012, Planet. Space Sci., 73, 98, doi: $10.1016 /$ j.pss.2012.03.009

Chambers, K. C., Magnier, E. A., Metcalfe, N., et al. 2016, arXiv e-prints, arXiv:1612.05560. https://arxiv.org/abs/1612.05560

Chesley, S. R., Farnocchia, D., Nolan, M. C., et al. 2014, Icarus, 235, 5, doi: 10.1016/j.icarus.2014.02.020

Christensen, E., Africano, B., Farneth, G., et al. 2018, in AAS/Division for Planetary Sciences Meeting Abstracts \#50, AAS/Division for Planetary Sciences Meeting Abstracts, 310.10

Clark, D. L., Spurný, P., Wiegert, P., et al. 2016, Astronomical Journal, 151, 135

DeMeo, F. E., Binzel, R. P., Slivan, S. M., \& Bus, S. J. 2009, Icarus, 202, 160

DeMeo, F. E., \& Carry, B. 2013, Icarus, 226, 723

DeMeo, F. E., \& Carry, B. 2014, Nature, 505, 629, doi: $10.1038 /$ nature 12908

Farnocchia, D., Chesley, S., \& Micheli, M. 2015a, Icarus, 258, 18

Farnocchia, D., Chesley, S. R., \& Chamberlin, A. B. 2016, in AAS/Division for Planetary Sciences Meeting Abstracts \#48, AAS/Division for Planetary Sciences Meeting Abstracts, 305.03

Farnocchia, D., Chesley, S. R., Milani, A., Gronchi, G. F., \& Chodas, P. W. 2015b, in Asteroids IV, ed. P. Michel, F. DeMeo, \& W. F. Bottke, 815-834, doi: 10.2458/azu_uapress_9780816532131-ch041 
Farnocchia, D., Eggl, S., Chodas, P. W., Giorgini, J. D., \& Chesley, S. R. 2019, Celestial Mechanics and Dynamical Astronomy, 131, 36, doi: 10.1007/s10569-019-9914-4

Farnocchia, D., Tholen, D. J., Micheli, M., et al. 2017, in AAS/Division for Planetary Sciences Meeting Abstracts \#49, AAS/Division for Planetary Sciences Meeting Abstracts, 100.09

Fedorets, G., Granvik, M., \& Jedicke, R. 2017, Icarus, 285, 83

Fedorets, G., Granvik, M., Jones, R. L., Jurić, M., \& Jedicke, R. 2020, Icarus, 338, 113517

Flewelling, H. A., Magnier, E. A., Chambers, K. C., et al. 2016, arXiv e-prints, arXiv:1612.05243.

https://arxiv.org/abs/1612.05243

Fukugita, M., Ichikawa, T., Gunn, J. E., et al. 1996, AJ, 111, 1748, doi: 10.1086/117915

Gaia Collaboration, Brown, A. G. A., Vallenari, A., Prusti, T., \& et al. 2018, A\&A, 616, A1, doi: 10.1051/0004-6361/201833051

Gaia Collaboration, Prusti, T., de Bruijne, J. H. J., et al. 2016, A\&A, 595, A1

Gehrels, T. 1967, The Astronomical Journal, 72, 929

Graham, M. J., Kulkarni, S. R., Bellm, E. C., et al. 2019, PASP, 131, 078001, doi: 10.1088/1538-3873/ab006c

Granvik, M., Jedicke, R., Bolin, B., et al. 2013, in Asteroids. Prospective Energy and Material Resources, ed. V. Badescu (Springer), $151-167$

Granvik, M., Vaubaillon, J., \& Jedicke, R. 2012, Icarus, 218, 262

Granvik, M., Morbidelli, A., Jedicke, R., et al. 2016, Nature, 530, 303

Granvik, M., Morbidelli, A., Jedicke, R., et al. 2018, Icarus, 312, 181, doi: 10.1016/j.icarus.2018.04.018

Harris, A. W., \& D'Abramo, G. 2015, Icarus, 257, 302

Hirase, Y., Nakamura, A. M., \& Michikami, T. 2004, Planetary and Space Science, 52, 1103

Hook, I. M., Jørgensen, I., Allington-Smith, J. R., et al. 2004, PASP, 116, 425, doi: 10.1086/383624

Ivezić, Ž., Kahn, S. M., Tyson, J. A., \& 311 authors. 2019, The Astrophysical Journal, 873, 111

Jedicke, R., Bolin, B. T., Bottke, W. F., et al. 2018a, Frontiers in Astronomy and Space Sciences, 5, A13

Jedicke, R., Sercel, J., Gillis-Davis, J., Morenz, K. J., \& Gertsch, L. 2018b, Planet. Space Sci., 159, 28, doi: 10.1016/j.pss.2018.04.005

Jenniskens, P., Shaddad, M. H., Numan, D., et al. 2009, Nature, 458, 485, doi: 10.1038/nature07920

Jenniskens, P., Albers, J., Koop, M. W., et al. 2016, in AIAA Science and Technology Forum and Exposition
Jordi, C., Gebran, M., Carrasco, J. M., et al. 2010, A\&A, 523, A48, doi: 10.1051/0004-6361/201015441

Kwiatkowski, T., Kryszczyńska, A., Polińska, M., et al. 2009, Astronomy \& Astrophysics, 495, 967

Lindegren, L., Hernández, J., Bombrun, A., et al. 2018, A\&A, 616, A2, doi: 10.1051/0004-6361/201832727

Mainzer, A., Masiero, J., Grav, T., et al. 2012, ApJ, 745, 7, doi: $10.1088 / 0004-637 \mathrm{X} / 745 / 1 / 7$

Marsden, B. 1969, The Astronomical Journal, 74, 720

Marsden, B., Sekanina, Z., \& Yeomans, D. K. 1973, The Astronomical Journal, 78, 211

Masci, F. J., Laher, R. R., Rusholme, B., et al. 2019, PASP, 131, 018003, doi: 10.1088/1538-3873/aae8ac

Melosh, H. J. 1985, Geology, 13, 144

Melosh, H. J. 1989, Impact cratering : a geologic process (Oxford University Press, Oxford, UK)

Micheli, M., Tholen, D. J., \& Elliott, G. T. 2012, New Astronomy, 17, 446

—. 2013, Icarus, 226, 251

—. 2014, The Astrophysical Journal Letters, 788, L1

Miles, R. 2011, Journal of British Astronomical

Association, 121, 350

Minor Planet Center. 2020, Minor Planet Electronic Circular 2020-D104

Mommert, M. 2017, Astronomy and Computing, 18, 47, doi: 10.1016/j.ascom.2016.11.002

Mommert, M., Hora, J. L., Farnocchia, D., et al. 2014a, ApJ, 786, 148, doi: 10.1088/0004-637X/786/2/148

Mommert, M., Farnocchia, D., Hora, J. L., et al. 2014b, ApJ, 789, L22, doi: 10.1088/2041-8205/789/1/L22

Muinonen, K., Belskaya, I. N., Cellino, A., et al. 2010, Icarus, 209, 542, doi: 10.1016/j.icarus.2010.04.003

Penttilä, A., Shevchenko, V. G., Wilkman, O., \& Muinonen, K. 2016, Planet. Space Sci., 123, 117, doi: $10.1016 /$ j.pss.2015.08.010

Pravec, P., \& Harris, A. W. 2007, Icarus, 190, 250, doi: $10.1016 /$ j.icarus.2007.02.023

Rabinowitz, D., Helin, E., Lawrence, K., \& Pravdo, S. 2000, Nature, 403, 165

Saito, J., Miyamoto, H., Nakamura, R., et al. 2006, Science, 312, 1341, doi: 10.1126/science. 1125722

Seidelmann, P. K., Abalakin, V. K., Bursa, M., et al. 2002, Celestial Mechanics and Dynamical Astronomy, 82, 83

Shevchenko, V. G., Belskaya, I. N., Muinonen, K., et al. 2016, Planetary and Space Science, 123, 101 , doi: https://doi.org/10.1016/j.pss.2015.11.007

Shober, P. M., Jansen-Sturgeon, T., Sansom, E. K., et al. 2019, The Astronomical Journal, 158, 183

Speyerer, E. J., Povilaitis, R. Z., Robinson, M. S., Thomas, P. C., \& Wagner, R. W. 2016, Nature, 538, 215 
Stetson, P. B. 1987, Publications of the Astronomical of the Pacific, 99, 191

Tody, D. 1986, in Society of Photo-Optical Instrumentation Engineers (SPIE) Conference Series, Vol. 627, Instrumentation in astronomy VI, ed. D. L. Crawford, 733, doi: 10.1117/12.968154

Tody, D. 1993, in Astronomical Society of the Pacific Conference Series, Vol. 52, Astronomical Data Analysis Software and Systems II, ed. R. J. Hanisch, R. J. V. Brissenden, \& J. Barnes, 173

Tricarico, P. 2017, Icarus, 284, 416
Vokrouhlický, D. 1998, Astronomy \& Astrophysics, 335, 1093

Vokrouhlický, D., \& Milani, A. 2000, A\&A, 362, 746

Warren, P. H. 1994, Icarus, 111, 338 , doi: https://doi.org/10.1006/icar.1994.1149

Xilouris, E. M., Bonanos, A. Z., Bellas-Velidis, I., et al. 2018, A\&A, 619, A141, doi: 10.1051/0004-6361/201833499

Zhao, H., Yao, J., \& Lu, H. 2007, Proceedings of the International Astronomical Union, 3, 565 


\section{APPENDIX}

Table 1. Telescopes used in this work and their purpose.

\begin{tabular}{|l|l|l|l|l|} 
Telescope & Aperture $(\mathrm{m})$ & Astrometry & Photometry & Lightcurve \\
\hline CSS Mt. Lemmon & 1.5 & $\checkmark$ & & \\
Calar Alto Schmidt & 0.8 & $\checkmark$ & & \\
Nordic Optical Telescope & 2.5 & $\checkmark$ & $\checkmark$ & \\
Gemini North & 8.1 & & \\
Canada-France-Hawai'i Telescope & 3.6 & $\checkmark$ & $\checkmark$ \\
Lowell Discovery Telescope & 4.3 & $\checkmark$ &
\end{tabular}

Table 2. Newly acquired or remeasured astrometric data for $2020 \mathrm{CD}_{3}$. The columns are, in order from left to right: observation date (UTC); right ascension; declination; Gaia system magnitude (Jordi et al. 2010); MPC observatory code; right ascension uncertainty in arcseconds; declination uncertainty in arcseconds; telescope name.

\begin{tabular}{|c|c|c|c|c|c|c|c|}
\hline Date (UTC) & $\alpha$ & $\delta$ & G & Code & $\sigma_{\alpha}$ & $\sigma_{\delta}$ & Telescope \\
\hline 20200215.526427 & 130337.570 & +091738.40 & 19.6 & G96 & 0.11 & 0.16 & CSS Mt. Lemmon \\
\hline 20200217.975500 & 134524.910 & +191844.71 & 21.0 & Z84 & 0.30 & 0.30 & Calar Alto Schmidt \\
\hline 20200217.984601 & 134526.960 & +192034.07 & 20.9 & $\mathrm{Z} 84$ & 0.32 & 0.32 & Calar Alto Schmidt \\
\hline 20200217.993701 & 134528.601 & +1922 22.81 & 21.1 & Z84 & 0.21 & 0.21 & Calar Alto Schmidt \\
\hline 20200218.002805 & 134529.872 & +192410.44 & 21.0 & Z84 & 0.22 & 0.22 & Calar Alto Schmidt \\
\hline 20200218.011907 & 134530.718 & +192557.10 & 21.3 & $\mathrm{Z} 84$ & 0.17 & 0.17 & Calar Alto Schmidt \\
\hline 20200218.021008 & 134531.196 & +192742.04 & 21.3 & $\mathrm{Z} 84$ & 0.19 & 0.19 & Calar Alto Schmidt \\
\hline 20200221.093805 & 140350.575 & +242051.66 & 21.8 & $\mathrm{Z} 84$ & 0.24 & 0.24 & Calar Alto Schmidt \\
\hline 20200221.114761 & 140344.360 & +242247.25 & 21.8 & Z84 & 0.27 & 0.27 & Calar Alto Schmidt \\
\hline 20200221.177717 & 140322.794 & +242737.51 & 21.8 & Z84 & 0.23 & 0.23 & Calar Alto Schmidt \\
\hline 20200221.198677 & 140315.400 & +242853.86 & 21.7 & Z84 & 0.15 & 0.15 & Calar Alto Schmidt \\
\hline 20200221.174711 & 140352.507 & +243107.79 & 22.0 & $\mathrm{Z} 23$ & 0.13 & 0.15 & NOT \\
\hline 20200221.199832 & 140342.272 & +243304.85 & 21.6 & $\mathrm{Z} 23$ & 0.10 & 0.12 & NOT \\
\hline 20200221.203876 & 140340.597 & +243322.12 & 21.6 & $\mathrm{Z} 23$ & 0.07 & 0.07 & NOT \\
\hline 20200224.586055 & 141438.369 & +272839.86 & 22.6 & 568 & 0.038 & 0.036 & Gemini North \\
\hline 20200224.601986 & 141431.235 & +272919.63 & 22.9 & 568 & 0.053 & 0.054 & Gemini North \\
\hline 20200224.608418 & 141428.325 & +272934.13 & 22.8 & 568 & 0.034 & 0.033 & Gemini North \\
\hline 20200224.621399 & 141422.582 & +273000.12 & 22.4 & 568 & 0.107 & 0.041 & Gemini North \\
\hline 20200225.148888 & 141623.887 & +274614.71 & 23.6 & $\mathrm{Z} 23$ & 0.2 & 0.2 & NOT \\
\hline 20200225.156760 & 141621.045 & +274642.49 & 22.7 & $\mathrm{Z} 23$ & 0.08 & 0.08 & NOT \\
\hline 20200225.161406 & 141619.302 & +274658.27 & 22.7 & $\mathrm{Z} 23$ & 0.11 & 0.11 & NOT \\
\hline 20200226.608875 & 141835.299 & +284016.90 & 23.0 & 568 & 0.047 & 0.041 & $\mathrm{UH} 2.2 \mathrm{~m}$ \\
\hline 20200226.613020 & 141833.500 & +284023.38 & 23.0 & 568 & 0.060 & 0.049 & $\mathrm{UH} 2.2 \mathrm{~m}$ \\
\hline 20200226.616949 & 141831.802 & +284029.28 & 23.2 & 568 & 0.075 & 0.078 & UH $2.2 \mathrm{~m}$ \\
\hline 20200226.620998 & 141830.056 & +284034.90 & 23.2 & 568 & 0.057 & 0.049 & $\mathrm{UH} 2.2 \mathrm{~m}$ \\
\hline 20200226.625629 & 141828.070 & +284040.98 & 23.0 & 568 & 0.055 & 0.045 & $\mathrm{UH} 2.2 \mathrm{~m}$ \\
\hline 20200226.620384 & 141830.334 & +284034.01 & 22.76 & 568 & 0.05 & 0.05 & CFHT \\
\hline
\end{tabular}




\begin{tabular}{|c|c|c|c|c|c|c|c|}
\hline \multicolumn{8}{|c|}{ Appendix Table 2 continued } \\
\hline Date (UTC) & $\alpha$ & $\delta$ & G & Code & $\sigma_{\alpha}$ & $\sigma_{\delta}$ & Telescope \\
\hline 20200226.621564 & 141829.827 & +284035.61 & 22.77 & 568 & 0.05 & 0.05 & CFHT \\
\hline 20200226.622763 & 141829.317 & +284037.19 & 22.74 & 568 & 0.05 & 0.05 & CFHT \\
\hline 20200228.594550 & 142141.875 & +293630.86 & 22.77 & 568 & 0.05 & 0.05 & CFHT \\
\hline 20200228.595729 & 142141.374 & +293632.58 & 23.19 & 568 & 0.05 & 0.05 & CFHT \\
\hline 20200228.598103 & 142140.361 & +293636.06 & 23.23 & 568 & 0.05 & 0.05 & CFHT \\
\hline 20200301.477536 & 142344.902 & +30 1525.54 & 22.6 & G37 & 0.256 & 0.268 & LDT \\
\hline 20200301.479237 & 142344.291 & +30 1527.50 & 23.0 & G37 & 0.313 & 0.283 & LDT \\
\hline 20200301.480279 & 142343.932 & +301528.52 & 22.8 & G37 & 0.213 & 0.262 & LDT \\
\hline 20200302.162378 & 142445.080 & +303046.94 & 23.2 & Z23 & 0.07 & 0.07 & NOT \\
\hline 20200302.179266 & 142438.827 & +3031 16.90 & 23.1 & Z23 & 0.09 & 0.09 & NOT \\
\hline 20200302.655003 & 142420.964 & +304213.62 & 23.32 & 568 & 0.05 & 0.05 & CFHT \\
\hline 20200302.656878 & 142420.269 & +304213.26 & 23.08 & 568 & 0.05 & 0.05 & CFHT \\
\hline 20200302.658754 & 142419.596 & +304212.91 & 23.13 & 568 & 0.05 & 0.05 & CFHT \\
\hline 20200304.635008 & 142548.907 & +31 1521.90 & 23.30 & 568 & 0.05 & 0.05 & CFHT \\
\hline 20200304.636888 & 142548.213 & +311521.78 & 23.28 & 568 & 0.05 & 0.05 & CFHT \\
\hline 20200304.638761 & 142547.512 & +31 1521.58 & 23.28 & 568 & 0.05 & 0.05 & CFHT \\
\hline 20200305.489286 & 142626.653 & +312351.85 & 23.1 & G37 & 0.06 & 0.06 & LDT \\
\hline 20200305.490947 & 142626.093 & +312352.37 & 22.9 & G37 & 0.06 & 0.06 & LDT \\
\hline 20200305.492737 & 142625.495 & +312352.87 & 22.9 & G37 & 0.05 & 0.05 & LDT \\
\hline 20200306.240152 & 142641.654 & +313509.84 & 23.3 & Z23 & 0.06 & 0.06 & NOT \\
\hline 20200306.246311 & 142639.515 & +313509.81 & 23.7 & Z23 & 0.07 & 0.07 & NOT \\
\hline 20200306.252078 & 142637.524 & +313509.36 & 23.6 & Z23 & 0.06 & 0.06 & NOT \\
\hline 20200321.462672 & 142258.469 & +331541.15 & 23.77 & 568 & 0.05 & 0.05 & CFHT \\
\hline 20200321.464548 & 142257.785 & +331543.12 & 23.72 & 568 & 0.05 & 0.05 & CFHT \\
\hline 20200321.466429 & 142257.087 & +331545.00 & 23.59 & 568 & 0.05 & 0.05 & CFHT \\
\hline 20200325.199063 & 141734.589 & +330950.72 & 24.0 & Z23 & 0.12 & 0.12 & NOT \\
\hline 20200325.210295 & 141730.563 & +330939.14 & 24.2 & Z23 & 0.26 & 0.26 & NOT \\
\hline 20200325.219775 & 141727.296 & +330928.11 & 23.8 & $\mathrm{Z} 23$ & 0.10 & 0.10 & NOT \\
\hline 20200329.161561 & 141140.795 & +324619.67 & 23.9 & $\mathrm{Z} 23$ & 0.08 & 0.05 & NOT \\
\hline 20200329.171345 & 141137.179 & +324609.34 & 24.1 & Z23 & 0.09 & 0.06 & NOT \\
\hline 20200329.182286 & 141133.175 & +324556.44 & 24.0 & Z23 & 0.09 & 0.06 & NOT \\
\hline 20200330.376555 & 140954.750 & +323345.95 & 23.4 & G37 & 0.09 & 0.08 & LDT \\
\hline 20200330.379675 & 140953.606 & +323343.93 & 23.5 & G37 & 0.17 & 0.17 & LDT \\
\hline 20200417.072836 & 134135.761 & +263434.21 & 24.1 & Z23 & 0.089 & 0.102 & NOT \\
\hline 20200417.083259 & 134132.407 & +263413.83 & 24.3 & Z23 & 0.070 & 0.064 & $\mathrm{NOT}$ \\
\hline 20200417.093646 & 134129.074 & +263352.11 & 23.8 & Z23 & 0.207 & 0.207 & NOT \\
\hline 20200429.105118 & 132542.426 & +193324.57 & & Z23 & 0.25 & 0.25 & NOT \\
\hline 20200429.113261 & 132540.749 & +193300.44 & 24.3 & Z23 & 0.25 & 0.25 & NOT \\
\hline 20200515.936339 & 132125.566 & +073909.07 & 25.0 & Z23 & 0.15 & 0.15 & NOT \\
\hline 20200517.244907 & 132156.056 & +064412.63 & 24.5 & 568 & 0.197 & 0.152 & CFHT \\
\hline $202005 \quad 17.247704$ & 132155.726 & +06 4405.99 & 24.3 & 568 & 0.281 & 0.272 & CFHT \\
\hline 20200520.950021 & 132311.161 & +040613.56 & 25.6 & $\mathrm{Z} 23$ & 0.10 & 0.10 & NOT \\
\hline 20200520.967845 & 132309.104 & +040528.13 & 25.4 & Z23 & 0.10 & 0.10 & NOT \\
\hline 20200520.985665 & 132307.102 & +040442.33 & 25.4 & $\mathrm{Z} 23$ & 0.10 & 0.10 & NOT \\
\hline
\end{tabular}


Table 3. Gemini North photometry of $2020 \mathrm{CD}_{3}$. The columns are, from left to right: sequential number of observation; filter; derived magnitude; instrumental error; zero-point error.

\begin{tabular}{|l|l|l|l|l|} 
Obs. id & Filter & Mag. & $\sigma_{I N S T}$ & $\sigma_{Z P}$ \\
\hline 1 & $r^{\prime}$ & 22.399 & 0.037 & 0.048 \\
2 & $i^{\prime}$ & 22.269 & 0.039 & 0.054 \\
3 & $r^{\prime}$ & 22.413 & 0.035 & 0.048 \\
4 & $g^{\prime}$ & 23.111 & 0.049 & 0.033 \\
5 & $i^{\prime}$ & 22.267 & 0.050 & 0.054 \\
6 & $r^{\prime}$ & 22.389 & 0.040 & 0.048 \\
7 & $g^{\prime}$ & 23.265 & 0.056 & 0.033 \\
8 & $i^{\prime}$ & 22.340 & 0.053 & 0.054
\end{tabular}

Table 4. Lightcurve photometric data for $2020 \mathrm{CD}_{3}$ obtained with the LDT translated to the $r_{\mathrm{P} 1}$ filter. The columns are, from left to right: observation date; measured magnitude; zero-point error; instrumental error.

\begin{tabular}{|l|l|l|l|} 
Date (MJD) & $\operatorname{Mag}\left(r_{\mathrm{P} 1}\right)$ & $\sigma_{Z P}$ & $\sigma_{I N S T}$ \\
\hline 58906.4881610 & 22.7116 & 0.0228 & 0.1087 \\
58906.4889677 & 22.6461 & 0.0215 & 0.1050 \\
58906.4894715 & 22.8355 & 0.0224 & 0.1289 \\
58906.4899752 & 22.6235 & 0.0227 & 0.1126 \\
58906.4905406 & 22.9247 & 0.0216 & 0.1407 \\
58906.4910444 & 22.8095 & 0.0227 & 0.1270 \\
58906.4915481 & 23.0082 & 0.0226 & 0.1508 \\
58906.4923176 & 22.8036 & 0.0218 & 0.1241 \\
58906.4928214 & 22.9557 & 0.0227 & 0.1618 \\
58906.4933251 & 22.6341 & 0.0213 & 0.1121 \\
58906.4938292 & 23.1323 & 0.0232 & 0.1768 \\
58906.4943329 & 22.8583 & 0.0225 & 0.1346 \\
58906.4953404 & 22.7895 & 0.0231 & 0.1333 \\
58906.4963479 & 22.8472 & 0.0229 & 0.1373 \\
58906.4968516 & 23.1161 & 0.0243 & 0.1807 \\
58906.4973554 & 23.1744 & 0.0229 & 0.1949 \\
58906.5013856 & 22.9624 & 0.0229 & 0.1503 \\
58906.5018894 & 22.9546 & 0.0232 & 0.1508 \\
58906.5029427 & 22.8884 & 0.0239 & 0.1488 \\
58906.5034464 & 22.6851 & 0.0234 & 0.1236 \\
58906.5039502 & 22.9444 & 0.0226 & 0.1473 \\
58906.5049578 & 23.1792 & 0.0225 & 0.1871 \\
58906.5054615 & 23.0686 & 0.0220 & 0.1513 \\
58906.5059652 & 22.9580 & 0.0224 & 0.1486 \\
58906.5074792 & 22.7658 & 0.0227 & 0.1207 \\
58906.5079829 & 22.9301 & 0.0223 & 0.1403 \\
58906.5089904 & 22.9475 & 0.0224 & 0.1535 \\
58906.5094942 & 22.6765 & 0.0228 & 0.1160 \\
58906.5099979 & 22.8687 & 0.0225 & 0.1325 \\
58906.5105017 & 23.0201 & 0.0225 & 0.1490
\end{tabular}

Appendix Table 4 continued on next page 
Appendix Table 4 continued

\begin{tabular}{|l|l|l|l|} 
Date (MJD) & $\operatorname{Mag}\left(r_{\mathrm{P} 1}\right)$ & $\sigma_{Z P}$ & $\sigma_{I N S T}$ \\
\hline 58906.5110060 & 22.6124 & 0.0221 & 0.1041 \\
58906.5120135 & 22.6595 & 0.0231 & 0.1040 \\
58906.5126895 & 23.1251 & 0.0223 & 0.1675 \\
58906.5131933 & 22.6779 & 0.0220 & 0.1112 \\
58906.5136970 & 22.9829 & 0.0231 & 0.1546 \\
58906.5142007 & 22.6173 & 0.0221 & 0.1030 \\
58906.5147052 & 22.9908 & 0.0232 & 0.1551 \\
58906.5152090 & 22.6400 & 0.0225 & 0.1069 \\
58906.5157131 & 23.0546 & 0.0228 & 0.1527 \\
58906.5162168 & 22.6199 & 0.0219 & 0.1141 \\
58906.5167207 & 22.6704 & 0.0226 & 0.1151 \\
58906.5172244 & 23.1026 & 0.0226 & 0.1690 \\
58906.5177281 & 22.6366 & 0.0217 & 0.1102 \\
58906.5182319 & 22.8375 & 0.0225 & 0.1326 \\
58906.5187356 & 22.8540 & 0.0232 & 0.1375 \\
58906.5192395 & 22.8399 & 0.0220 & 0.1317 \\
58906.5197432 & 22.6965 & 0.0221 & 0.1144 \\
58906.5202471 & 23.0981 & 0.0220 & 0.1653 \\
58906.5207509 & 22.7457 & 0.0214 & 0.1193 \\
58906.5212546 & 22.8318 & 0.0218 & 0.1292 \\
58906.5217587 & 23.0903 & 0.0221 & 0.1685 \\
58906.5222624 & 22.7996 & 0.0216 & 0.1359 \\
58906.5228400 & 22.6896 & 0.0227 & 0.1138 \\
58906.5233449 & 22.9262 & 0.0230 & 0.1470 \\
58906.5238487 & 23.1095 & 0.0217 & 0.1704 \\
58906.5243527 & 22.7774 & 0.0224 & 0.1202 \\
58906.5248564 & 22.7482 & 0.0228 & 0.1234 \\
58906.5253602 & 22.7669 & 0.0222 & 0.1259 \\
58906.5258639 & 22.9694 & 0.0228 & 0.1472 \\
58906.5263677 & 22.7342 & 0.0225 & 0.1164 \\
58906.5283826 & 23.1254 & 0.0226 & 0.1831 \\
58906.5288865 & 22.8278 & 0.0224 & 0.1341 \\
58906.5293902 & 22.7815 & 0.0221 & 0.1421 \\
58906.5298940 & 22.7310 & 0.0239 & 0.1272 \\
58906.5319088 & 22.7927 & 0.0230 & 0.1459
\end{tabular}


Table 5. Fits of the $H, G_{1}, G_{2}$ system to the photometric phase curve of $2020 \mathrm{CD}_{3}$. The first five fits assume values for the $G_{1}$ and $G_{2}$ parameters typical for the spectral types mentioned in the parenthesis and fit for $H$ only. The last fit allows for both $H$ and $G_{12}$ parameters to be fitted, but requires $0<G_{12}<1$, which is a physically meaningful range. The last two columns provide the weighted root-mean-square (wRMS) value and the Bayesian Information Criterion with respect to its lowest value $(\Delta \mathrm{BIC})$. These results have been computed with the online calculator available at http://h152.it.helsinki.fi/HG1G2/.

\begin{tabular}{llllll} 
Fit type & $H_{V}$ & $G_{1}$ & $G_{2}$ & wRMS & $\Delta$ BIC \\
\hline$H(\mathrm{E})$ & 32.13 & 0.1505 & 0.6005 & 1.632 & 0.000 \\
$H(\mathrm{~S} / \mathrm{M})$ & 31.79 & 0.2588 & 0.3721 & 1.635 & 0.4107 \\
$H(\mathrm{P})$ & 31.63 & 0.8343 & 0.04887 & 1.658 & 3.310 \\
$H(\mathrm{C})$ & 31.54 & 0.8228 & 0.01938 & 1.661 & 3.686 \\
$H(\mathrm{D})$ & 31.69 & 0.9617 & 0.01645 & 1.661 & 3.763 \\
$H, G_{12}$ & 31.88 & 0.000 & 0.5324 & 1.630 & 4.418 \\
\hline
\end{tabular}

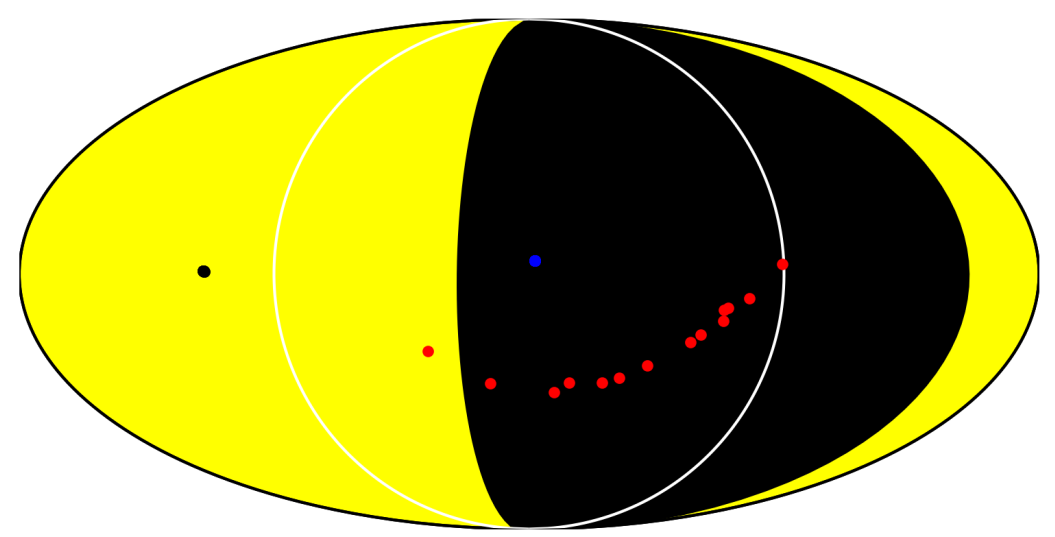

Figure 6. Mollweide projection of the surface of the Moon on 2017 September 15. Yellow and black regions show the day and night sides of the Moon, respectively. Red points show the locations from where the $2020 \mathrm{CD}_{3}$ samples from the lunar origin hypothesis are ejected. Blue and black points show the sub-Earth and sub-solar points corresponding to the sample ejection times. White circle encloses the area of the Moon visible from Earth. 